\title{
Pathological and molecular examination findings in postmortem testis biopsies reveal spermatogenesis damages in COVID-19 patients
}

\section{Xixiang Ma}

Huazhong University of Science and Technology

\section{Chuhuai Guan}

Huazhong University of Science and Technology

\section{Rong Chen}

Wuhan Jinyintan Hospital

\section{Yunyun Wang}

Huazhong University of Science and Technology

\section{Shenglei Feng}

Huazhong University of Science and Technology

\section{rongshuai wang}

Department of Forensic Medicine, Tongji Medical College, Huazhong University of Science and

Technology

\section{Guoqiang Qu}

Hubei Chongxin Judicial Expertise Center

\section{Liling Wang}

Huazhong University of Science and Technology

\section{Sijia Zhao}

Huazhong University of Science and Technology

\section{Fengli Wang}

Huazhong University of Science and Technology

\section{Xiaoli Wang}

Huazhong University of Science and Technology

\section{Dingyu Zhang}

Wuhan Jinyintan Hospital

\section{Liang Liu}

Huazhong University of Science and Technology

\section{Aihua Liao}

Huazhong University of Science and Technology

https://orcid.org/0000-0001-8533-8315

\section{Shuiqiao Yuan ( $\nabla$ shuiqiaoyuan@hust.edu.cn )}


Article

Keywords:

Posted Date: August 19th, 2020

DOI: https://doi.org/10.21203/rs.3.rs-56526/v1

License: (c) (i) This work is licensed under a Creative Commons Attribution 4.0 International License. Read Full License 
1 Pathological and molecular examination findings in postmortem testis biopsies

reveal spermatogenesis damages in COVID-19 patients

4 Xixiang Ma ${ }^{1 a}$, Chuhuai Guan ${ }^{2 a}$, Rong Chen ${ }^{3 a}$, Yunyun Wang ${ }^{2}$, Shenglei Feng ${ }^{1}$, Rongshuai

5 Wang $^{4}$, Guoqiang $\mathrm{Qu}^{4}$, Liling Wang ${ }^{1}$, Sijia Zhao ${ }^{1}$, Fengli Wang ${ }^{1}$, Xiaoli Wang ${ }^{1}$, Dingyu

6 Zhang ${ }^{5,6}$, Liang Liu ${ }^{2 *}$, Aihua Liao ${ }^{1 *}$, Shuiqiao Yuan ${ }^{1 *}$

$8{ }^{1}$ Institute of Reproductive Health, Tongji Medical College, Huazhong University of Science

9 and Technology, Wuhan, Hubei 430030, China

$10{ }^{2}$ Department of Forensic Medicine, Tongji Medical College, Huazhong University of Science

11 and Technology, Wuhan, Hubei 430030, China

$12{ }^{3}$ Department of Pathology, Wuhan Jinyintan Hospital, Wuhan, Hubei 430023, China

$13{ }^{4}$ Hubei Chongxin Judicial Expertise Center, Wuhan, Hubei 430415, China

$14{ }^{5}$ Research Center for Translational Medicine, Wuhan Jinyintan Hospital, Wuhan, Hubei

15 430023, China

$16{ }^{6}$ Joint Laboratory of Infectious Diseases and Health, Wuhan Institute of Virology and

17 Wuhan Jinyintan Hospital, Chinese Academy of Sciences, Wuhan, Hubei 430023 China

${ }^{\text {a }}$ Authors contributed equally to the manuscript.

\section{${ }^{*}$ Corresponding authors}

21 Liang Liu, M.D., Department of Forensic Medicine, Tongji Medical College, Huazhong 22 University of Science and Technology, Wuhan, Hubei 430030, China. Phone: +86- 02723 83692644; Email: 907505@qq.com

24 Aihua Liao, M.D., Institute of Reproductive Health, Tongji Medical College, Huazhong 25 University of Science and Technology, Wuhan, Hubei 430030, China. Phone: +8626 15994262573; Email: aihua liao@sina.com

27 Shuiqiao Yuan, Ph.D., Institute of Reproductive Health, Tongji Medical College, Huazhong 28 University of Science and Technology, Wuhan, Hubei 430030, China. +86-15527507840; 29 Email: shuiqiaoyuan@hust.edu.cn 


\section{Abstract}

32 Despite widespread interest in the pathophysiology of COVID-19 in respiratory and

33 cardiovascular systems, little is known about the morphologic and molecular changes in the

34 testis of patients with COVID-19 and the effects of SARS-CoV-2 infection on male fertility.

35 We report here on the pathophysiology and molecular feature of testes obtained at autopsy

36 from six men with COVID-19, as compared with those of testes from three men with age-

37 matched, uninfected SARS-CoV-2. Our histopathological results showed that all COVID-19

38 patients had severe spermatogenesis damages compared with controls. Importantly, we

39 detected the nuclear acid of the SARS-CoV-2 virus, viral particles, and SARS-CoV-2 spike

40 S1 protein in COVID-19 patient testes, and we also found ACE2 and TMPRSS2

41 significantly elevated in the testes from COVID-19 patients. Furthermore, we observed a

42 prominent leukocyte infiltration, including $\mathrm{CD}^{+} \mathrm{T}$ lymphocytes, $\mathrm{CD}^{+} \mathrm{O}^{+} \mathrm{B}$ lymphocytes,

$43 \mathrm{CD}^{+} 8^{+}$macrophages, HLA-DR ${ }^{+}$myeloid cells, and $\mathrm{CD}^{+}$plasma cells in the testes of

44 COVID-19 patients. RNA-Seq analyses further revealed SARS-CoV-2 infection could lead

45 to dysfunction of the genes that regulate the spermatogenesis and inflammation response-

46 related pathways. Collectively, our pathological and molecular examination findings indicate

47 that SARS-CoV-2 could directly attack testicular cells, thereby inducing the damage of

48 testicular immune privilege and spermatogenesis defects.

51 Keywords: COVID-19, spermatogenesis, autopsy, male fertility, SARS-CoV-2

53 Running title: The SARS-CoV-2 infection leads to spermatogenesis disorder 
Introduction

In late December 2019, a novel coronavirus (severe acute respiratory syndrome

58 coronavirus 2, SARS-CoV-2) has emerged in Wuhan, China and its subsequent rapid

59 international spread pose a global health emergency, which causing pneumonia symptoms

60 (COVID-19) in humans similar to those caused by SARS coronavirus (SARS-CoV). Unlike

61 the mild threaten of SARS-CoV, SARS-CoV-2 having influential infectious and leading to

62 over 10 million people infected, and more than 499,913 patients died of COVID-19 around

63 the world by June 29, 2020 (Coronavirus disease (COVID-19) Situation Report-161, World

64 Health Organization, https://www.who.int/emergencies/diseases/novel-coronavirus-

$652019 /$ situation-reports). With the increasing number of people infected with COVID-19

66 across the world, the scientific community began to study the pathophysiology of the SARS-

67 Cov-2 virus to understand a new, emergent, and unknown pathogen and to guide disease

68 transmission, prevention, susceptibility, and treatment. Although an autopsy can provide an

69 opportunity for us to understand the potential key information about the real mechanisms

70 underlying death and organ damage caused by COVID-19 infections(Salerno et al., 2020),

71 there is a lack of well-defined pathophysiology examination of different organs from the

72 patients who died of COVID-19 disease. Since progressive respiratory failure is the primary

73 cause of death in the COVID-19 pandemic, researchers mainly focused on the

74 pathophysiological changes in major organs at autopsy, such as the lung, heart, and Kidney

75 (Ackermann et al., 2020; Fox et al., 2020; Liu et al., 2020; Schaller et al., 2020; Su et al., 76 2020), whereas ignored the anatomical pathophysiology observation of other organs, 77 especially reproductive organs. Meanwhile, millions of people with SARS-CoV-2 infections 78 have been cured, among them a large number of men who have fertility desire, which 79 raises concerns about whether SARS-CoV-2 could attack the testicular cells and might 80 cause male infertility. Two studies were unable to detect the presence of SARS-CoV-2 in 
81 semen and testis from men who had recovered from COVID-19 (Pan et al., 2020; Song et

82 al., 2020), but in contrast, a recent study has reported that SARS-CoV-2 can be detected in

83 semen of patients with COVID-19 ( $\mathrm{Li}$ et al., 2020). Therefore, it is currently unknown

84 whether the infection of SARS-CoV-2 will damage spermatogenesis and male fertility or

85 whether SARS-CoV-2 can attack the germ cell into seminiferous tubules.

86 We report here, for the first time to our knowledge, on the pathophysiology and

87 molecular feature of testes obtained at autopsy from six men with a recorded cause of

88 death of COVID-19, as compared with those of testes from three men who died from other

89 diseases and age-matched, uninfected control testes. Our pathological results showed that

90 all six male patients with COVID-19 had severe spermatogenesis damage compared with

91 controls, indicating SARS-CoV-2 infection impairs male germ cell development and

92 spermatogenesis. More importantly, the coronavirus particles were observed in the testis of

93 patients with COVID-19 under an electronic microscope, and the nuclear acid of the SARS-

94 CoV-2 virus was also detectable in COVID-19 patient testis sections, which indicates that

95 SARS-CoV-2 could enter the testicular tubules and attack the testicular cells. At the

96 molecular level, we found that the mRNA and protein levels of ACE2 and TMPRSS2 (the

97 SARS-CoV-2 entry receptor and spike protein priming, respectively) appear to be

98 overexpressed in formalin-fixed paraffin-embedded (FFPE) testis sections from COVID-19

99 patients. We also detected the SARS-CoV-2 spike S1 signals in the seminiferous tubules of

100 patients with COVID-19, which further demonstrate SARS-CoV-2 attack the germ cells via

101 binding its receptors. In addition, we observed a prominent infiltration of leukocytes,

102 including $\mathrm{CD}^{+} \mathrm{T}$ lymphocytes, $\mathrm{CD}^{+} 8^{+}$macrophages, and HLA-DR ${ }^{+}$myeloid cells in the

103 testes of COVID-19 patients. Most importantly, under normal conditions, $\mathrm{CD}^{+} \mathrm{B}^{+} \mathrm{B}$

104 lymphocytes and $\mathrm{CD}^{+} 8^{+}$plasma cells are not present in human testes, whereas were

105 observed in testes of COVID-19 patients. RNA-seq analyses further revealed that genes 
106 involved in spermatogenesis and some inflammation response-related pathways in the

107 testis of patients with COVID-19 were deregulated, which provided a possible molecular

108 explanation for the inflammatory immune attack and spermatogenesis damages in testis

109 caused by SARS-CoV-2 infection. Taken together, our pathological and molecular

110 examination findings at autopsy provide the most substantial direct evidence and

111 underlying molecular mechanism for the effects of SARS-CoV-2 infection on

112 spermatogenesis and male fertility and are likely to have important implications for the

113 reproductive treatment of COVID-19 in this male patient population.

115 Results and discussion

116 To assess the effects of SARS-CoV-19 infection on spermatogenesis and male germ

117 development, we first performed a PAS-staining assay to examine the testicular

118 morphology in all patients with COVID-19. Numerous degenerated germ cells (GC) could

119 be observed to fall off to the lumen in seminiferous tubules of all six COVID-19 patients (Fig.

1201 and Fig.S1A). In contrast, the germ cells did not fall off and aligned well in the whole

121 seminiferous tubules of three control patients (Fig. 1). Strikingly, in five of six cases, the

122 changes of germ cells lost were massive, with only a few germ cells left and attached the

123 seminiferous tubules (patient-1, 3, 4, 5, and 6). In particular, many seminiferous tubules in

124 the testes of patients 4 and 5 are barely saw the intact germ cells but only present Sertoli

125 cells (SCs), similar to Sertoli cell-only syndrome. In agreement with this morphological

126 observation, the number of DDX4 (a germ cell marker) positive cells was dramatically

127 reduced in all testicular specimens from the COVID-19 groups (Fig. 2A). Interestingly, the

128 number of Sertoli cells was comparable in the testes from SARS-CoV-2 infected patients

129 and uninfected patients (Fig. 2B), suggesting that SARS-CoV-2 infection may not impair

130 Sertoli cell development. These morphological changes in testes of COVID-19 patients 
131 indicate that SARS-CoV-2 infection could impair the male germ cell development and

132 eventually lead to germ cells lost. It is worth noting that the patient-6 was recovered from

133 COVID-19 after nine days of antiviral treatment and died of massive gastrointestinal

134 bleeding caused by liver cirrhosis at home after the discharge of 45 days (Table S1). The

135 main reason we included the patient-6 in this study was to observe whether there was any

136 damage in the spermatogenesis of recovered patients with COVID-19 after treatment.

137 Strikingly, the testes of patient-6 also showed severe spermatogenesis damage (Fig.S1A),

138 which prompted us to pay more attention to the spermatogenesis and fertility of the

139 recovered patients from SARS-CoV-2 infection. However, we could not rule out the

140 possibility of effects of chronic hepatitis B viral infection and liver cirrhosis on the

141 spermatogenesis in the patient-6 because chronic hepatitis B infection increases the

142 incidence of apoptosis and necrosis in spermatogenic cells(Moretti et al., 2008).

143 To ascertain how the male germ cell loss in the patients with COVID-19, we asked

144 whether the apoptosis and inflammation reaction occurred in the testicular cells of COVID-

14519 patients. TUNEL assay revealed that the number of apoptotic cells in COVID-19 testes

146 was significantly higher than the control testes (Fig.S1B-C), a finding consistent with the

147 phenotype of Zika virus infection in mice(Govero et al., 2016; Ma et al., 2016), raising a

148 possibility of SARS-CoV-2 damage the immune privilege and innate immune homeostasis

149 of the testis. Indeed, immunohistochemical (IHC) staining for various leukocytes revealed a

150 scattered infiltration of $\mathrm{CD}^{+} \mathrm{T}^{2}$ lymphocytes, $\mathrm{CD} 20^{+} \mathrm{B}$ lymphocytes, CD68 ${ }^{+}$macrophages,

151 and $\mathrm{HLA}-\mathrm{DRa}^{+}$myeloid cells were observed in seminiferous tubules of patients with

152 COVID-19, whereas rarely detected in controls (Fig. 3A-D). Besides, we found that CD38 ${ }^{+}$

153 cells (a marker of plasma cells) are highly observed in the interstitial compartments of

154 COVID-19 patient testes (Fig.3E). Correspondingly, extensive IgG precipitation was mainly

155 found in the interstitial compartments and partly in the seminiferous epithelium of COVID-19 
156 patient testes, which is consistent with the effects of SARS infected testes (Fig.3F)(Xu et al., 157 2006). These data indicate that SARS-CoV-2 could directly target testicular cells and

158 thereby cause testicular immune attack, leading to germ cell injury and lost eventually.

159 Furthermore, in the current study, the histological analysis showed exfoliation of the 160 spermatogenic epithelium and reduction of germ cells in all six COVID-19 patient testes, 161 especially the patients 4 and 5 exhibited thickening of the tubular wall and almost Sertoli 162 cell-only symptom (Fig.1). These severe damages in the testes are similar to orchitis 163 caused by some viruses, such as HIV and SARS, and leukocyte infiltration is a main 164 pathological feature of these viral orchitides (Liu et al., 2018; Xu et al., 2006). As it has 165 been known, no B cells reside in testes under physiological conditions. Thus, the evidence 166 of a prominent infiltration of leukocytes, specifically the infiltration of B cells and plasma 167 cells, and IgG precipitation observed in the testes of COVID-19 patients indicate that the 168 blood-testis barrier and the immune-privileged environment were damaged in COVID-19 169 patients. Like other viral orchitides(Xu et al., 2006), SARS-CoV-2 might trigger a secondary 170 autoimmune response and contribute to the primary pathogenesis of viral orchitis, which 171 accounts for the testicular damage.

172 To further determine whether the SARS-CoV-2 can attack testicular cells directly, we 173 first detected the nuclear acid of SARS-CoV-2 in formalin-fixed paraffin-embedded (FFPE) 174 testis tissues of COVID-19 patients. Through RT-qPCR-based virus nuclear acid detection, 175 we found that the two testis samples (patient-1 and 3) were positive for SARS-CoV-2 176 nuclear acid (Table S2). The detection of the SARS-CoV-2 virus within the testis, a finding 177 consistent with other study(Li et al., 2020), suggests that direct viral effects, as well as the 178 induced damage of the testicular immune privilege, may contribute to the germ cell injury. 179 Since the spike (S) glycoprotein of coronaviruses is known to be essential in the binding of 180 the SARS-CoV-2 to the host cells, we then examined the SARS-CoV-2 spike protein 
181 expression in the testes of patients with COVID-19 by immunohistochemistry using SARS-

182 CoV Spike S1 antibody. We found the positive signals of SARS-CoV Spike S1 are present

183 in all COVID-19 patient testicular sections (Fig.4A), suggesting that the SARS-CoV-2

184 indeed infected with the testicular cells through its spike glycoprotein binding mechanism. It

185 should note that we detected the SARS-CoV-2 Spike protein in all COVID-19 patient testes,

186 but the nuclear acid of SARS-CoV-2 was only detected in two samples (patient-1 and 3).

187 This difference may be due to the fact that viral RNA was extracted from FFPE samples,

188 which causing the viral RNA is easy to degrade and hard to detect by qPCR assay. Next,

189 we examined the testis sections with electron microscopy to directly see whether the

190 coronavirus particles were present in the testis of patients with COVID-19. We selected

191 three patient testes (Patient-1, 3, and 4) to perform electron microscopy analyses and found

192 that the coronavirus particles can be seen in the interstitial compartment of testes (Fig. 4B

193 and Fig.S1D). This electron microscopy data provide strong direct evidence for SARS-

194 CoV-2 to enter and attack human testicular tissues. Further studies are required with

195 respect to the detailed information about virus shedding, survival time, and concentration in

196 testis. Although spermatogenesis disruption was probably a common feature in the testes

197 from COVID-19 patients, we observed the different degrees of spermatogenesis damages

198 in patients with COVID-19. This may contribute to the relative number of copies of the virus

199 into the testis and the duration of the virus infection.

200 Since SARS- CoV-2 binds to the angiotensin-converting enzyme 2 (ACE2) receptor for 201 entry and the serine protease TMPRSS2 for spike protein priming to attack the 202 cells(Hoffmann et al., 2020; Lan et al., 2020), we next examined both protein and mRNA 203 levels of ACE2 and TMPRSS2 in testes by immunohistochemistry and RT-qPCR analysis, 204 respectively. Immunostainings revealed that although both ACE2 and TMPRSS2 proteins 205 were predominantly expressed in the cytoplasm and membrane of spermatocytes, 
206 spermatids, and Sertoli cells in the control testes, the elevated ACE2 and TMPRSS2 207 signals were observed in the seminiferous tubules of all patients with COVID-19 (Fig. 4C-D

208 and Fig. S2A-B). Interestingly, both ACE2 and TMPRSS2 proteins were not found to 209 express in spermatogonia in our study and one recent report (Fig.4C-D)(Yang et al., 2020).

210 In contrast, two previous studies by single-cell transcriptome database analyses revealed

211 that the ACE2 and TMPRSS2 mRNA were predominantly enriched in spermatogonia and

212 Sertoli cells(Pan et al., 2020; Wang and Xu, 2020). Consistent with the

213 immunohistochemistry result, RT-qPCR revealed the mRNA levels of ACE2 and TMPRSS2

214 significantly increased in all COVID-19 patient testes compared to that of control patient

215 testes (Fig. 4E-F). Remarkably, both the protein and mRNA levels of ACE2 and TMPRSS2

216 appeared to be a very significant increase in the testes of COVID-19 patients 4 and 5 who

217 had much severe spermatogenesis disruption (Fig. 1 and Fig. 4E-F). Given that the fact of

218 ACE2 and TMPRSS2 are integral membrane protein that appears to be the host-cell

219 receptor and spike protein priming for SARS-CoV-2 to enter the cells(Hoffmann et al., 2020;

220 Lan et al., 2020), the increased expression of ACE2 and TMPRSS2 may facilitate infection

221 with COVID-19 and increase the risk of cellular damage by SARS-CoV-2(Fang et al., 2020).

222 In line with this notion, our data showed the significantly higher signal intensity of ACE2-

223 and TMPRSS2-positive cells in the testes from patients with COVID-19 than in those from

224 uninfected controls, which explained the underlying reasons for the SARS-CoV-2 attack

225 testicular cells.

226 To investigate the impact and molecular changes of SARS-CoV-2 infections in testes, 227 we next extracted total RNA from testes of controls (control-1, 2, and 3) and three COVID22819 patients (patient-2, 3, and 4), respectively, and performed RNA-seq to analyze the 229 transcriptome changes. With regard to the coding and IncRNA genes, RNA-seq analyses 230 found 28,801 expressed genes from control and COVID-19 patient testes (Table S3), of 
231 which 2,645 genes up-regulated and 2,789 genes down-regulated in COVID-19 patients 232 compared with controls (fold of change>2, FDR<0.001) (Fig. 5A, Fig. S3A, and Table S4).

233 These results suggest that SARS-CoV-2 infection could trigger the transcriptome dynamic

234 alterations at molecular levels in testes during specific biological processes.

235 To further determine the changes of transcriptome upon SARS-CoV-2 infection in 236 testes, we performed Gene Ontology (GO) term analysis for those differentially regulated 237 genes. Consistent with our virus RNA detection and immunohistochemical results, GO 238 analysis demonstrated that up-regulated transcripts were significantly enriched in the 239 invasion of the virus, such as "viral gene expression" and "viral life cycle" (e.g., RPL13, 240 VTA1, RPS27, WWP1, XPO1, and VAPA) (Fig.5B). Importantly, some inflammation-related 241 processes were activated, including "interleukin-6-mediated signaling pathway" and 242 "regulation of B cell proliferation" (e.g., IL6ST, IL2, MEF2C, PTPRC, BCL2, and BCL6) 243 (Fig.5B). Additionally, GO enrichment documented that up-regulated genes were 244 significantly enriched in autophagy and response to oxygen levels as well (Fig.5B). 245 Consistent with our testis histological results (Fig.1), the down-regulated genes significantly 246 enriched in "spermatogenesis" and "reproduction" (Fig.5C), which further illustrated the 247 impact of SARS-CoV-2 infection on male fertility. Strikingly, in line with our inflammatory cell 248 immunostaining results (Fig.3), we found some genes (e.g., BCR, BCRP1, and BCRP2) 249 that were down-regulated in which involved in some inflammation-related processes and 250 immune response, such as "negative regulation of myeloid leukocyte mediated immunity", 251 "negative regulation of leukocyte degranulation and migration", and "neutrophil activation 252 involved in immune response" (Fig.5C). We further determined the effect of SARS-CoV-2 253 infection on alternative splicing (AS) events of genes from testes of COVID-19 patients by 254 RNA-seq analyses. Compare to deregulated genes, and we scored only a few significantly 255 altered AS events in testes of COVID-19 patients (224 events with $P$-value $<0.05$ and 
$256|\Delta \psi|>0.1)$ (Fig.S3B and Table S5). However, GO analysis revealed the deregulated genes

257 with significant AS events enriched in virus-associated terms, such as "IRES-dependent

258 viral translational initiation" and "viral RNA genome replication" (Fig.5D). In addition, some

259 AS genes were enriched in inflammation-related NF-kappaB signaling, "regulation of I-

260 kappaB kinase/NF-kappaB signaling" and "NF-kappaB import into nucleus" (Fig.5D). These

261 bioinformatics data suggest that SARS-CoV-2 infection could lead to dysfunction of the

262 genes that regulate the spermatogenesis and inflammation-related pathways, thereby

263 causing inflammatory immune attack in testes and spermatogenesis defects.

264 Furthermore, since previous clinical reports indicate that SARS-CoV-2 infection can

265 cause inflammatory cytokine storm syndrome in COVID-19 patients(Gao et al., 2020;

266 Huang et al., 2020), we asked whether the inflammatory cytokines significantly elevated in

267 the testes of COVID-19 patients. Through analyses of our RNA-seq data, we found that 32

268 Inflammatory cytokines were up-regulated considerably with $P$-value $<0.05$ and fold of

269 change>2 in COVID-19 patient testes. There were 10 cytokines (CMTM6, FAM3C, INHBA,

270 IL33, TNFSF10, NAMPT, CMTM4, CCL28, IL2, and TIMP1) significantly up-regulated in

271 testes of COVID-19 patients when adjusting FDR<0.001 (Fig. 5E). Taken together, our data

272 indicate that the testis, despite its immune-privileged status, cannot be isolated from the

273 whole immune system upon infection of SARS-CoV-2, thereby inducing immune cells can

274 directly or indirectly target the testicular cells to promote spermatogenesis damage. The

275 RNA-Seq data further support the observation in the testes of SARS-CoV-2 infected

276 patients that inflammatory cytokines can activate the autoimmune response (Fig.3),

277 destroying the seminiferous epithelium (Fig.1), which is similar with SARS infected men

278 that were showing autoimmune orchitis(Xu et al., 2006). Besides, it should be noted that

279 high levels of cytokines following viral infection, illness, or injury can cause deterioration in 
280 spermatogenesis and steroidogenesis(Hedger and Meinhardt, 2003), thus adversely

281 affecting fertility, as all COVID-19 patients had other underlying diseases (Table S1).

282 Nevertheless, this study has limitations, including the small number of cases from a

283 single-center, and the patients were older people, which may not represent the fecundity of

284 men in the golden age of childbearing. For example, the patients with COVID-19 were

285 examined in this study are all aging men and had comorbidities, which may compromise

286 spermatogenesis and male germ cell development(Almeida et al., 2017; Shiraishi and

287 Matsuyama, 2018). Nonetheless, our analysis suggests that this possibility is unlikely,

288 particularly since the degree of germ cell apoptosis in the patients with COVID-19 increased

289 significantly, whereas in the control patients (also aging men), it remained stable at a

290 significantly lower level. Although our sample was small, 5 out of 6 cases showed a very

291 severely disrupted spermatogenesis feature in COVID-19 patients (Fig.1 and Fig.S1A),

292 which indicates that, to no small extent, SARS-CoV-2 infection does damage the human

293 reproductive system, especially spermatogenesis and male fertility.

294 Collectively, our findings provide direct evidence and underlying molecular mechanism

295 of SARS-CoV-2 for entry testis and attack the germ cells, which enhances the

296 understanding of COVID-19 pandemic in spermatogenesis and male fertility. Our findings

297 also suggest that researches should be undertaken to find ways to mitigate the risk of

298 testicular cell damage to protect male fertility during the COVID-19 course and treatment.

299 However, the relationship of these findings to the clinical course of COVID-19 and the in-

300 depth mechanism of SARS-CoV-2 infect testicular cells requires further study to elucidate.

301 Further research is still urgently needed for a comprehensive understanding of COVID-19

302 on male reproductive health, including effects on the viral sex transmission and testicular

303 endocrine function. 
305 Materials and methods

306 Patient selection and brief clinical summary

307 We analyzed autopsy specimens from five male patients who died from respiratory failure 308 caused by SARS-CoV-2 infection and compared them with testes from three uninfected 309 control males who died from other diseases (control-1 died with hemorrhagic shock, 310 control-2 died with coronary disease, and control-3 died with traffic accident). The COVID31119 patients were aged $51-83$ with mean $( \pm S D)$ ages of $69 \pm 11.5$ years, and the uninfected

312 control patients were aged $71-80$ with mean ages of $76 \pm 4.7$ years. All patients with COVID-

31319 had at least one common underlying disease, the most common of which were 314 hypertension (detailed patient characteristics in Table S1). All the COVID-19 patients were 315 confirmed SARS-CoV-2 positive by RT-qPCR and bilateral ground-glass opacities by Chest 316 X-rays according to the New Coronavirus Pneumonia Prevention and Control Program (7th 317 edition). Treatment in the ICU initially included antiviral drugs, antibiotics, glucocorticoid, 318 and antihypertensive drugs for all patients. The study was approved by and conducted 319 according to requirements of the ethics committees at Wuhan Jinyintan Hospital and Tongji

320 Medical College, Huazhong University of Science and Technology. Written informed 321 consent was obtained from the next of kin of each enrolled case.

\section{Histological analysis}

324 Testes were obtained at autopsy and fixed in a $10 \%$ formaldehyde solution. Then the 325 samples were processed to paraffin blocks according to the standard procedure. $5 \mu \mathrm{m}$ 326 sections were cut and stained with periodic acid-Schiff (PAS) after being dewaxed and 327 rehydrated. 
330 Apoptotic cells were detected by TUNEL assay using TUNEL Apoptosis Detection Kit

331 (C1098, Beyotime Biotechnology, Inc., Beijing, China) according to the manufacturer's

332 protocols. In brief, after dewaxing and rehydration, the testicular sections were incubated

333 for $15 \mathrm{~min}$ in $20 \mathrm{mg} / \mathrm{mL}$ Proteinase $\mathrm{K}$ (in PBS) solution. Then, the sections were covered

334 with $3 \% \mathrm{H} 2 \mathrm{O} 2$ and incubated at room temperature for $15 \mathrm{~min}$, followed by a TdT-labeled

335 reaction mixture with an enzyme, and avoid light incubation at $37^{\circ} \mathrm{C}$ for $1 \mathrm{~h}$. The sections

336 were rinsed with PBS between incubations at room temperature with a stop solution for 10

337 min. Finally, Streptavidin-HRP was added at room temperature for $30 \mathrm{~min}$. After another

338 rinse with PBS, the sections were incubated for color reaction with DAB solution at room

339 temperature for 5-10 min. Dehydrate using graded ethanol, vitrification by dimethylbenzene.

340 Images were obtained with an Axio Scope A1 microscope (Zeiss, Germany).

342 Immunohistochemistry

343 After being dewaxed and rehydrated, the $5 \mu \mathrm{m}$ thick sections were microwaved in $0.01 \mathrm{M}$

344 sodium citrate buffer $(\mathrm{pH}=6.0)$ for antigen retrieval, then cooled down to room temperature.

345 The sections were washed in PBS three times (10min per time), and endogenous

346 peroxidase activity was blocked with $3 \% \mathrm{H}_{2} \mathrm{O}_{2}$ in PBS. The sections were blocked in

347 blocking solution (containing $3 \%$ normal goat serum and $3 \%$ fetal bovine serum in $1 \%$

348 bovine serum albumin) for $1 \mathrm{~h}$ and were incubated with primary antibodies, including

349 primary antibodies against ACE2 (dilution $5 \mu \mathrm{g} / \mathrm{mL} ;$ AF933; R\&D Systems), TMPRSS2

350 (dilution 1:1000; ab92323; Abcam), DDX4 (dilution 1:200; ab13840; Abcam), SOX9 (dilution

351 1:1000; EPR14335-78; Abcam), CD3 (dilution 1:200; 17617-1-AP; Proteintech), CD20

352 (dilution 1:10000; 60271-1-Ig; Proteintech), CD68 (dilution 1:1000; 28058-1-AP;

353 Proteintech), HLA-DRA (dilution 1:300; 17221-1-AP; Proteintech), CD38 (dilution 1:200;

354 25284-1-AP; Proteintech), human IgG (dilution 1:10000; 10284-1-AP; Proteintech), SARS- 
355 CoV-2 Spike antibody (dilution 1:200; 40150-T62-COV2; Sino Biological) in a humidified

356 box overnight at $4^{\circ} \mathrm{C}$. After three times washes in PBS, sections were then incubated with

357 peroxidase-labeled secondary antibody (dilution 1:1000; Rabbit Anti-Goat IgG, A21030;

358 Goat Anti-Mouse IgG, A21010; Goat Anti-Rabbit IgG, A21020; Abbkine) for 1h at room

359 temperature and counterstained with hematoxylin. Images were photographed with an Axio

360 Scope A1 microscope (Zeiss, Germany).

362 RNA isolation and RT-qPCR for SARS-CoV-2 virus

363 Total RNA was extracted from deparaffinized formalin-fixed paraffin-embedded (FFPE)

364 sections using RNA RNeasy FFPE Kit (Qiagen, Germany) according to the manufacturer's

365 protocols. Virus quantification in the FFPR sample was performed using the COVID-19 366 (ASRS-CoV-2) Nucleic Acid Test Kit (Easy Diagnosis Biomedicine Co., Ltd, Wuhan, China) 367 with primers/probes for RNaseP and ORF1ab genes amplification following the Kit 368 instruction. Two-step real-time PCR was run on the StepOnePlus ${ }^{\mathrm{TM}}$ Software system 369 (ThermoFisher, USA). PCR running conditions were as follows: $50^{\circ} \mathrm{C}$ for $15 \mathrm{~min}, 95^{\circ} \mathrm{C}$ for 370 the $30 \mathrm{~s}, 40$ cycles at $95^{\circ} \mathrm{C}$ for $3 \mathrm{~s}, 60^{\circ} \mathrm{C}$ for $40 \mathrm{~s}$. The amplification curve was exponential, 371 ORF1ab with CT value less than 38 , and RNaseP with CT value less than 35 considered as 372 positive.

\section{RNA library preparation and sequencing}

375 Total RNAs were extracted from deparaffinized FFPE sections of controls (control-1, 2, and 3) and three COVID-19 patients (patient-2, 3, and 4) using RNA RNeasy FFPE Kit (Qiagen,

377 Germany) according to manufacturer's protocol. The RNA concentration was verified using 378 a NanoDrop 2000 Spectrophotometer (Thermo Fisher Scientific Inc). RNA finally quantified 379 by Qubit3.0 with QubitTM RNA Broad Range Assay kit (Life Technologies, Q10210). 
For RNA library preparation, $2 \mu \mathrm{g}$ of total RNA was used from each sample to prepare 381 RNA sequencing library using Ribo-off rRNA Depletion Kit (Human/Mouse/Rat) (Cat. 382 MRZG12324, Illumina) and KC-DigitalTM Stranded mRNA Library Prep Kit for Illumina® 383 (Cat. DR08502, Wuhan Seqhealth Co., Ltd. China) following the manufacturer's instruction.

384 The kit eliminates duplication bias in PCR and sequencing steps, by using a unique 385 molecular identifier (UMI) of 8 random bases to label the pre-amplified cDNA molecules. 386 The library products corresponding to $200-500$ bps were enriched, quantified, and finally 387 sequenced on NovaSeq 6000 sequencer (Illumina) with PE150 model.

389 RNA-Seq data analysis

390 Trimmomatic (version 0.36) was used to remove adaptor sequences, and low quality reads 391 from the sequencing data. Clean Reads were further treated with in-house scripts to

392 eliminate duplication bias introduced in library preparation and sequencing. De-duplicated 393 Reads were mapped to the reference genome of Homo sapiens from 394 ftp://ftp.ensembl.org/pub/release-87/fasta/homo_sapiens/dna/ using STRA software 395 (version 2.5.3a) with default parameters. Reads mapped to the exon regions of each gene 396 were counted by featureCounts (Subread-1.5.1; Bioconductor), and then RPKM was 397 calculated. Genes differentially expressed between groups was performed by the edgeR 398 package (version 3.12.1). The differential expressed genes were set with the threshold of 399 FDR $<0.001$ and fold change $\geq 2$. Alternative splicing events were detected by using rMATS 400 (version 3.2.5) with an FDR value cutoff of 0.05 and an absolute value of $\Delta \psi$ of 0.05 . Gene 401 ontology (GO) analyses for differentially expressed genes were implemented by KOBAS 402 software (version: 2.1.1) with a $P$-value cutoff of 0.05 to judge statistically significant 403 enrichment. 
406 All comparisons of numeric variables (including those in the gene-expression analysis) 407 were conducted with Student's $t$-test family-wise error rates. Original $P$ values are reported 408 only for the tests that met the criteria for false discovery rates. The results were presented 409 as mean $\pm \mathrm{SD}$, and significance was assumed for $P<0.05$. All the analyses were done 410 using SPSS, version 20 (IBM).

\section{Online supplemental material}

413 Fig. S1 shows the representative PAS-staining images of Patient-6 testes (A), TUNEL 414 assays for testicular sections of control patients and COVID-19 patients (B-C), and electron 415 microscopy image of the testis from Patient-3 (D). Fig. S2 presents the ACE2 and 416 TMPRSS2 immunohistochemical staining images in the testicular sections of all control 417 patients and COVID-19 patients. Fig.S3 shows the differential gene expression and 418 alternative splicing events in the testes of COVID-19 patients. Table S1 contains detailed 419 information on patient clinical characteristics and medical history in the study. Table S2 lists 420 the results of SARS-CoV-2 nuclear acid detection in FFPE testis samples of COVID-19 421 patients by RT-qPCR. Table S3 lists the total coding and IncRNA genes identified from the 422 testes of control and COVID-19 patients. Table S4 contains the up- and down-regulated

423 genes identified from testes of COVID-19 patients. Table S5 lists the alternative splicing 424 events identified from deregulated genes.

\section{Acknowledgments}

427 We thank the patients and their families for their dedication to help others understand this 428 disease. We are also grateful for the support of the clinical staff and technicians for 429 performing the autopsy work. 
431 Ferring Institute of Reproductive Medicine, Ferring Pharmaceuticals and Chinese Academy

432 of Sciences (FIRMSCOV02), the Fundamental Research Funds for the Central Universities

433 (2020kfyXGYJ116), the Cultivation Plan of International Joint Research Platform of

434 Huazhong University of Science and Technology (5001519009), and Emergency Research

435 Funds of Ministry of Science and Technology of P.R. China (2020YFC0844700).

436 Author contributions: SY and AL conceived and designed the experiments; LL, CG,

437 RC, YW, RG, GQ, and DZ conducted autopsy; XM, SF, LW, SZ, FW, and XW carried out

438 the testicular pathology experiments and contributed to data interpretation; XM and SF

439 performed the immunohistochemical experiments and RNA extraction; SY wrote the

440 manuscript. All authors have read and approved the final manuscript.

442 Disclosures: The authors declare no competing interests exist.

\section{References}

Ackermann, M., S.E. Verleden, M. Kuehnel, A. Haverich, T. Welte, F. Laenger, A. Vanstapel, C. Werlein, H. Stark, A. Tzankov, W.W. Li, V.W. Li, S.J. Mentzer, and D. Jonigk. 2020. Pulmonary Vascular Endothelialitis, Thrombosis, and Angiogenesis in Covid-19. The New England journal of medicine

Almeida, S., L. Rato, M. Sousa, M.G. Alves, and P.F. Oliveira. 2017. Fertility and Sperm Quality in the Aging Male. Curr Pharm Des 23:4429-4437.

Fang, L., G. Karakiulakis, and M. Roth. 2020. Are patients with hypertension and diabetes mellitus at increased risk for COVID-19 infection? The Lancet. Respiratory medicine 8:e21.

Fox, S.E., A. Akmatbekov, J.L. Harbert, G. Li, J. Quincy Brown, and R.S. Vander Heide. 2020. Pulmonary and cardiac pathology in African American patients with COVID-19: an autopsy series from New Orleans. The Lancet. Respiratory medicine

Gao, Y.M., G. Xu, B. Wang, and B.C. Liu. 2020. Cytokine storm syndrome in coronavirus disease 2019: A narrative review. Journal of internal medicine

Govero, J., P. Esakky, S.M. Scheaffer, E. Fernandez, A. Drury, D.J. Platt, M.J. Gorman, J.M. Richner, E.A. Caine, V. Salazar, K.H. Moley, and M.S. Diamond. 2016. Zika virus infection damages the testes in mice. Nature 540:438-442.

Hedger, M.P., and A. Meinhardt. 2003. Cytokines and the immune-testicular axis. Journal of reproductive immunology 58:1-26. 
Hoffmann, M., H. Kleine-Weber, S. Schroeder, N. Kruger, T. Herrler, S. Erichsen, T.S. Schiergens, G. Herrler, N.H. Wu, A. Nitsche, M.A. Muller, C. Drosten, and S. Pohlmann. 2020. SARSCoV-2 Cell Entry Depends on ACE2 and TMPRSS2 and Is Blocked by a Clinically Proven Protease Inhibitor. Cell 181:271-280 e278.

Huang, C., Y. Wang, X. Li, L. Ren, J. Zhao, Y. Hu, L. Zhang, G. Fan, J. Xu, X. Gu, Z. Cheng, T. Yu, J. Xia, Y. Wei, W. Wu, X. Xie, W. Yin, H. Li, M. Liu, Y. Xiao, H. Gao, L. Guo, J. Xie, G. Wang, R. Jiang, Z. Gao, Q. Jin, J. Wang, and B. Cao. 2020. Clinical features of patients infected with 2019 novel coronavirus in Wuhan, China. Lancet 395:497-506.

Lan, J., J. Ge, J. Yu, S. Shan, H. Zhou, S. Fan, Q. Zhang, X. Shi, Q. Wang, L. Zhang, and X. Wang. 2020. Structure of the SARS-CoV-2 spike receptor-binding domain bound to the ACE2 receptor. Nature 581:215-220.

Li, D., M. Jin, P. Bao, W. Zhao, and S. Zhang. 2020. Clinical Characteristics and Results of Semen Tests Among Men With Coronavirus Disease 2019. JAMA network open 3:e208292.

Liu, Q., R.S. Wang, G.Q. Qu, Y.Y. Wang, P. Liu, Y.Z. Zhu, G. Fei, L. Ren, Y.W. Zhou, and L. Liu. 2020. Gross examination report of a COVID-19 death autopsy. Fa yi xue za zhi 36:21-23.

Liu, W., R. Han, H. Wu, and D. Han. 2018. Viral threat to male fertility. Andrologia 50:e13140.

Ma, W., S. Li, S. Ma, L. Jia, F. Zhang, Y. Zhang, J. Zhang, G. Wong, S. Zhang, X. Lu, M. Liu, J. Yan, W. Li, C. Qin, D. Han, C. Qin, N. Wang, X. Li, and G.F. Gao. 2016. Zika Virus Causes Testis Damage and Leads to Male Infertility in Mice. Cell 167:1511-1524 e1510.

Moretti, E., M.G. Federico, V. Giannerini, and G. Collodel. 2008. Sperm ultrastructure and meiotic segregation in a group of patients with chronic hepatitis B and C. Andrologia 40:286-291.

Pan, F., X. Xiao, J. Guo, Y. Song, H. Li, D.P. Patel, A.M. Spivak, J.P. Alukal, X. Zhang, C. Xiong, P.S. Li, and J.M. Hotaling. 2020. No evidence of severe acute respiratory syndrome-coronavirus 2 in semen of males recovering from coronavirus disease 2019. Fertility and sterility 113:1135-1139.

Salerno, M., F. Sessa, A. Piscopo, A. Montana, M. Torrisi, F. Patane, P. Murabito, G.L. Volti, and C. Pomara. 2020. No Autopsies on COVID-19 Deaths: A Missed Opportunity and the Lockdown of Science. Journal of clinical medicine 9:

Schaller, T., K. Hirschbuhl, K. Burkhardt, G. Braun, M. Trepel, B. Markl, and R. Claus. 2020. Postmortem Examination of Patients With COVID-19. Jama

Shiraishi, K., and H. Matsuyama. 2018. Effects of medical comorbidity on male infertility and comorbidity treatment on spermatogenesis. Fertility and sterility 110:1006-1011 e1002.

Song, C., Y. Wang, W. Li, B. Hu, G. Chen, P. Xia, W. Wang, C. Li, F. Diao, Z. Hu, X. Yang, B. Yao, and Y. Liu. 2020. Absence of 2019 Novel Coronavirus in Semen and Testes of COVID-19 Patients. Biol Reprod

Su, H., M. Yang, C. Wan, L.X. Yi, F. Tang, H.Y. Zhu, F. Yi, H.C. Yang, A.B. Fogo, X. Nie, and C. Zhang. 2020. Renal histopathological analysis of 26 postmortem findings of patients with COVID-19 in China. Kidney international

Wang, Z., and X. Xu. 2020. scRNA-seq Profiling of Human Testes Reveals the Presence of the ACE2 Receptor, A Target for SARS-CoV-2 Infection in Spermatogonia, Leydig and Sertoli Cells. Cells 9:

Xu, J., L. Qi, X. Chi, J. Yang, X. Wei, E. Gong, S. Peh, and J. Gu. 2006. Orchitis: a complication of severe acute respiratory syndrome (SARS). Biol Reprod 74:410-416.

Yang, M., S. Chen, B. Huang, J.M. Zhong, H. Su, Y.J. Chen, Q. Cao, L. Ma, J. He, X.F. Li, X. Li, J.J. Zhou, J. Fan, D.J. Luo, X.N. Chang, K. Arkun, M. Zhou, and X. Nie. 2020. Pathological Findings in the Testes of COVID-19 Patients: Clinical Implications. European urology focus 
512 Figure legends
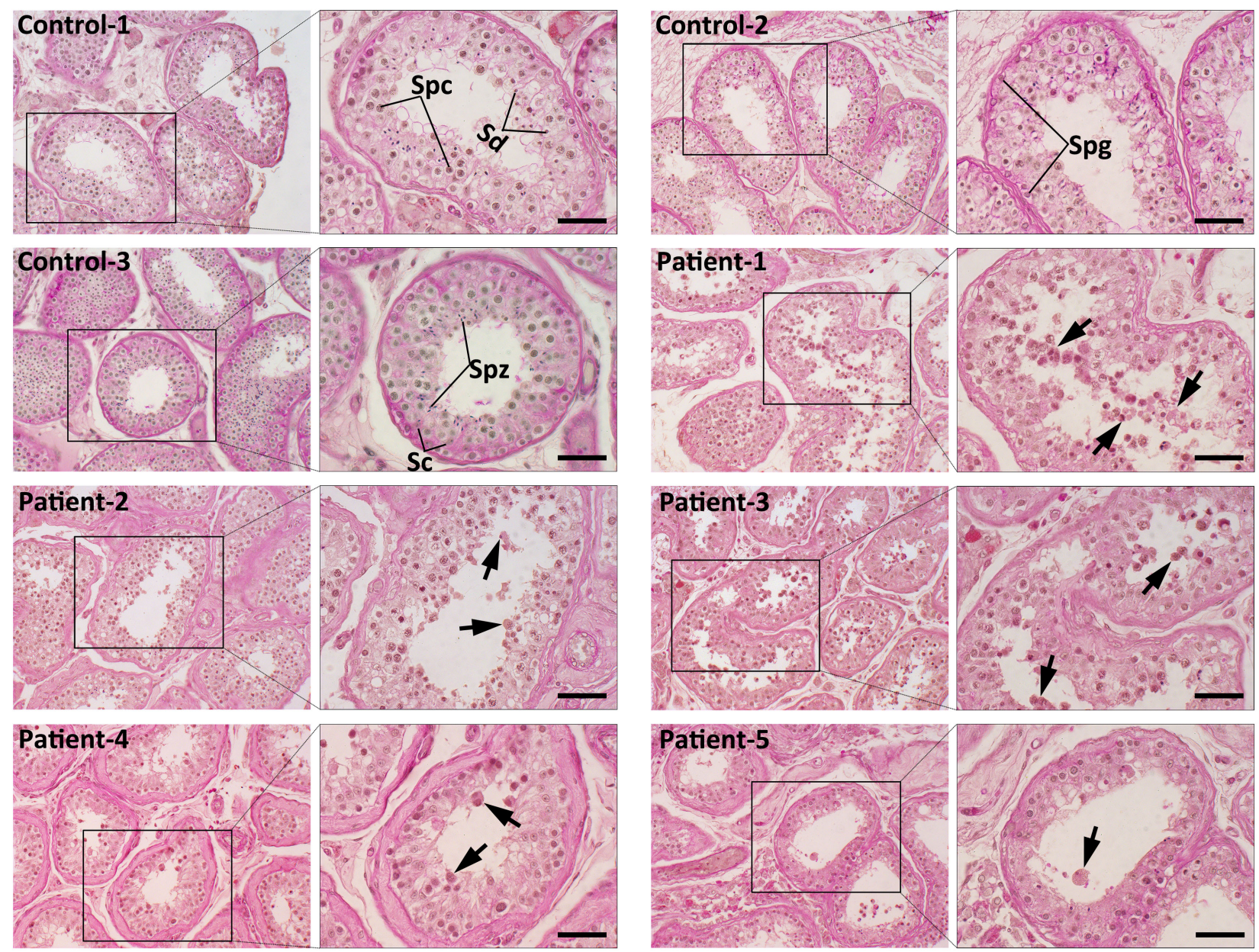

514 Figure 1. Spermatogenesis damages were observed in COVID-19 patients. Histological

515 analyses of the testicular sections from COVID-19 patients (patient-1, 2, 3, 4, and 5) and

516 uninfected control patients (control-1, 2, and 3) showing numerous degenerated germ cells

517 fell off to the lumen in seminiferous tubules of all five COVID-19 patients, whereas normal

518 spermatogenesis was observed in control patients. Spg, Spermatogonia; Spc,

519 Spermatocytes; Sd, Spermatids; Spz, Spermatozoa; Sc, Sertoli cells. Arrows indicate

520 degenerated germ cells. Scale bar=100 $\mu \mathrm{m}$.

521 
A

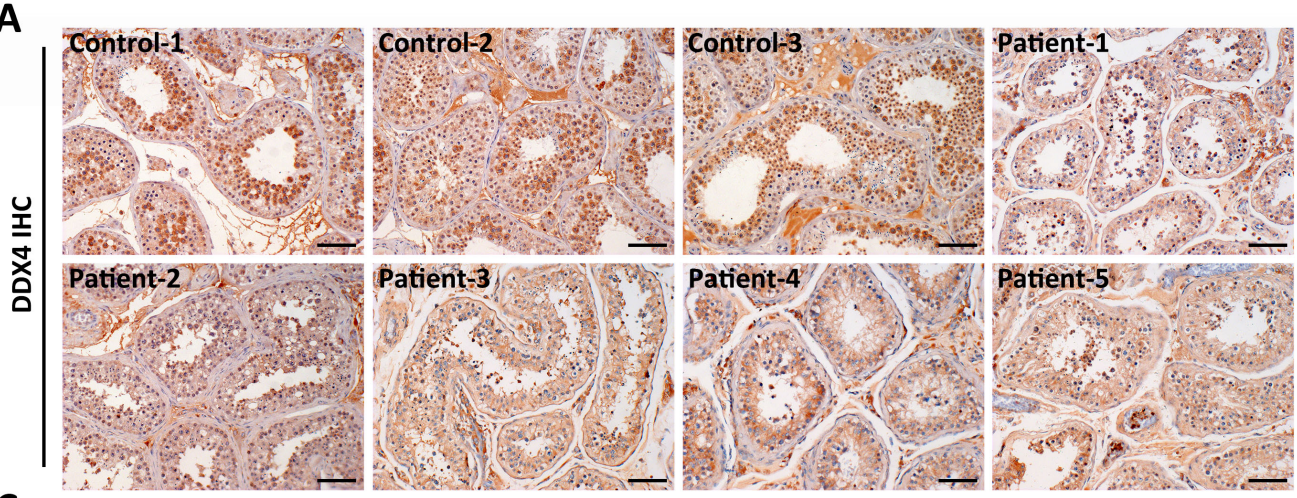

C

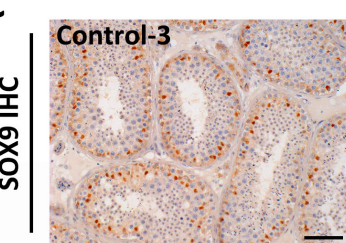

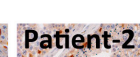

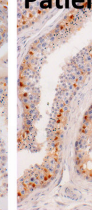

Patient-3

Patient-4

Figure 2. Germ cells but not Sertoli cells lost in the testes of COVID-19 patients. (A)

526 Immunohistochemical staining of DDX4 (a germ cell marker) in the testicular sections of

527 control patients (control-1, 2, and 3) and COVID-19 patients (patient-1, 2, 3, 4, and 5) is

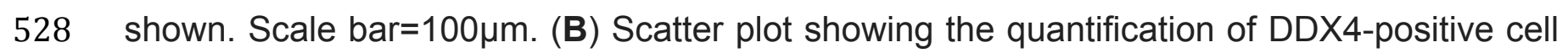

529 numbers per tubule in $(\mathrm{A}){ }^{* * *}, P<0.001$ by Student's $t$-test. $(\mathrm{C})$ Immunohistochemical

530 staining of SOX9 (a Sertoli cell marker) in the testicular sections of control patient (control-3)

531 and COVID-19 patients (patient-2, 3, and 4) is shown. Scale bar=100 $\mu$ m. (D) Scatter plot

532 showing the quantification of SOX9-positive cell numbers per tubule in (C). ns., not 533 significant. 

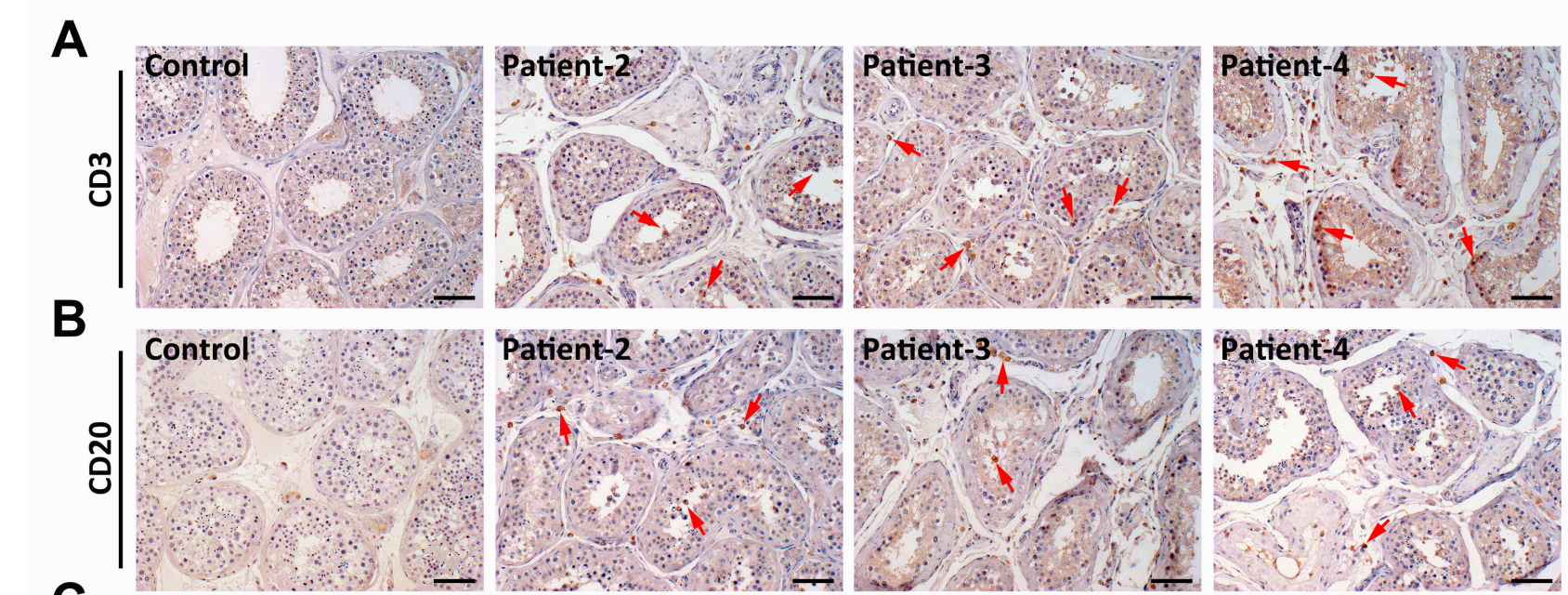

C
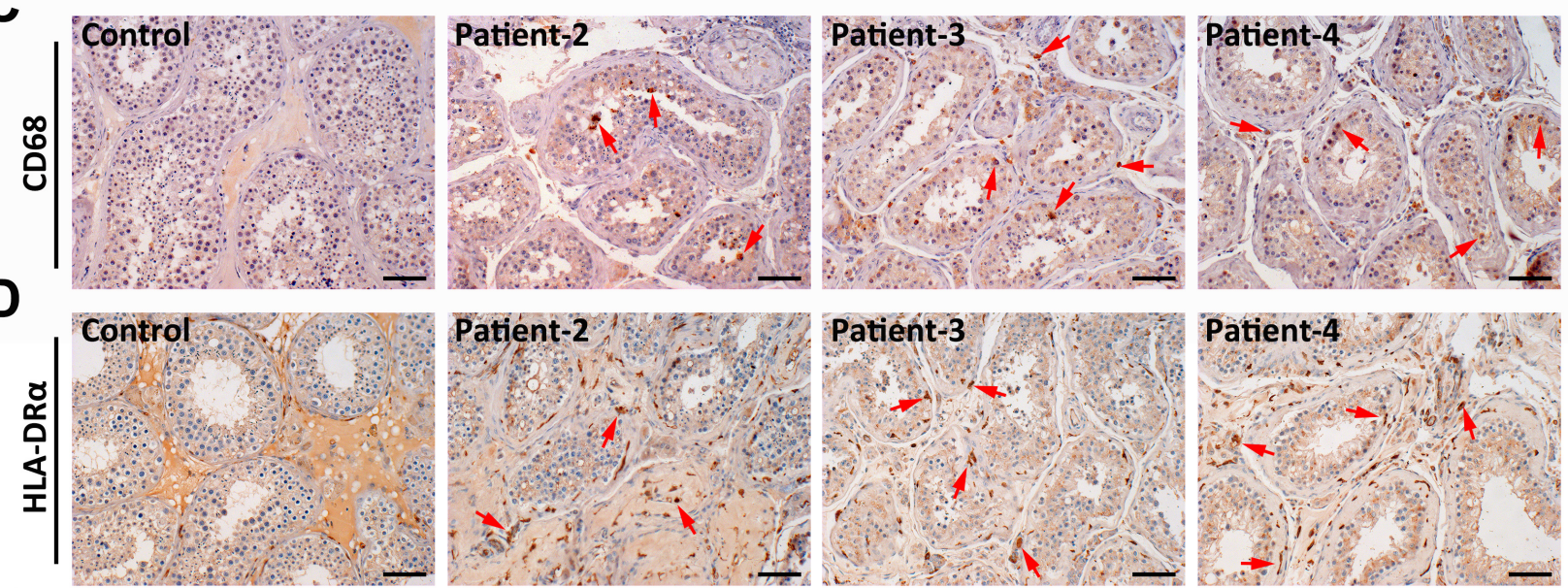

E
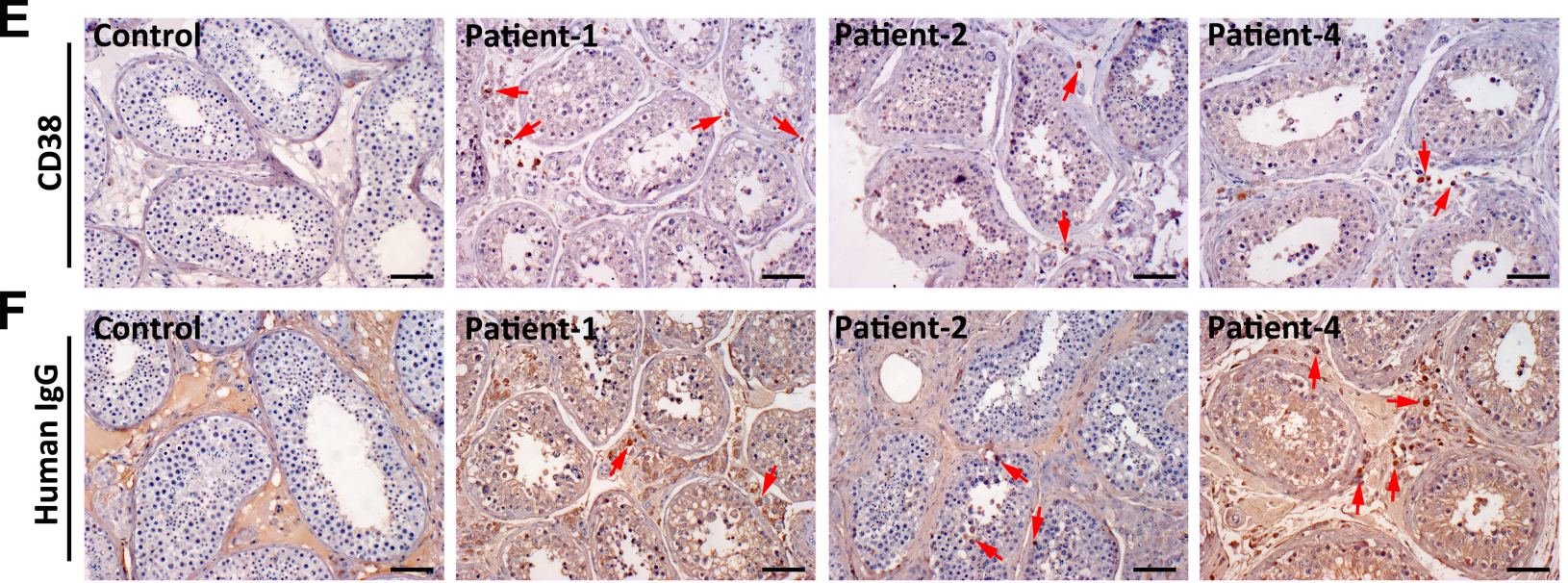

543 Figure 3. COVID-19 patient testes exhibit a prominent infiltration of various

544 inflammatory cells. (A) Representative CD3 immunohistochemical staining images in the

545 testicular sections of control and COVID-19 patients (patient-2, 3, and 4) are shown. Red

546 arrows indicate $\mathrm{CD}^{+} \mathrm{T}$ lymphocytes. Scale bar $=100 \mu \mathrm{m}$. (B) Representative CD20

547 immunohistochemical staining images in the testicular sections of control and COVID-19 
548 patients (patient-2, 3, and 4) are shown. Red arrows indicate CD20 ${ }^{+}$B lymphocytes. Scale 549 bar=100 $\mu$ m. (C) Representative CD68 immunohistochemical staining images in the 550 testicular sections of control and COVID-19 patients (patient-2, 3, and 4) are shown. Red

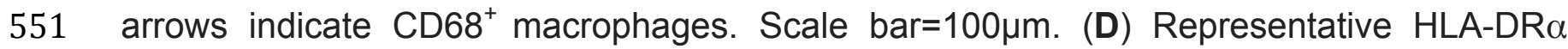
552 immunohistochemical staining images in the testicular sections of control and COVID-19 553 patients (patient-2, 3, and 4) are shown. Red arrows indicate HLA-DRA ${ }^{+}$myeloid cells. 554 Scale bar=100 $\mu$ m. (E) Representative CD38 immunohistochemical staining images in the 555 testicular sections of control and COVID-19 patients (patient-1, 2, and 4) are shown. Red

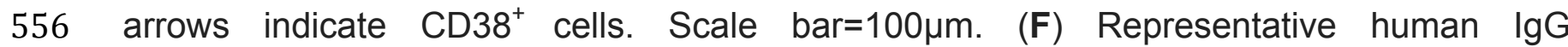
557 immunohistochemical staining images in the testicular sections of control and COVID-19 558 patients (patient-1, 2, and 4) are shown. Red arrows indicate IgG ${ }^{+}$cells. Scale bar=100 $\mu \mathrm{m}$. 559 

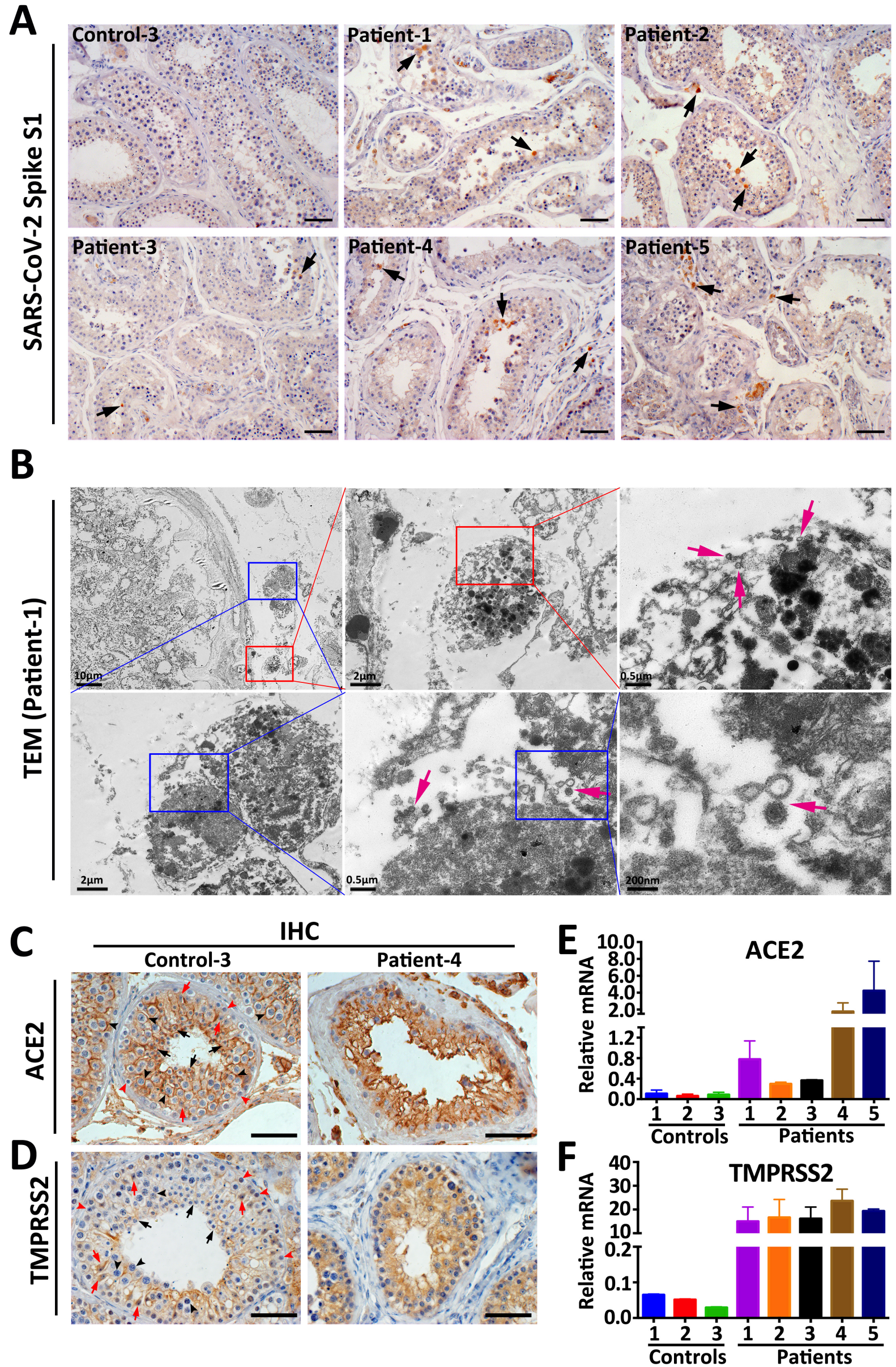
573 Figure 4. SARS-CoV-2 can invade testis and attack testicular cells. (A) Representative

574 SARS-CoV-2 Spike protein immunohistochemical staining images in the testicular sections

575 of control (control- 3) and COVID-19 patients (patient-1, 2, 3, 4, and 5) are shown. Black

576 arrows indicate SARS-CoV-2 Spike S1 positive cells. Scale bar=100 um. (B) Electron

577 microscopy of the testis from Patient-1, showing coronavirus particles suggestive of viral

578 infection (viral particles highlighted by red arrows). (C) Representative ACE2

579 immunohistochemical staining images in the testicular sections of control (control- 3) and

580 COVID-19 patient (patient-4) are shown. Black arrows indicate round spermatids; Black

581 arrowheads indicate pachytene spermatocytes; Red arrows indicate Sertoli cells; Red

582 arrowheads indicate spermatogonia. Scale bar=100 $\mu$ m. (D) Representative TMPRSS2

583 immunohistochemical staining images in the testicular sections of control (control- 3) and

584 COVID-19 patient (patient-4) are shown. Black arrows indicate round spermatids; Black

585 arrowheads indicate pachytene spermatocytes; Red arrows indicate Sertoli cells; Red

586 arrowheads indicate spermatogonia. Scale bar=100 $\mu \mathrm{m}$. (E) RT-qPCR analyses of the

587 relative ACE2 mRNA levels in control patients (control-1, 2, and 3) and COVID-19 patients

588 (patient-1, 2, 3, 4, and 5). (F) RT-qPCR analyses of the relative TMPRSS2 mRNA levels in 589 control patients (control-1, 2, and 3) and COVID-19 patients (patient-1, 2, 3, 4, and 5). 

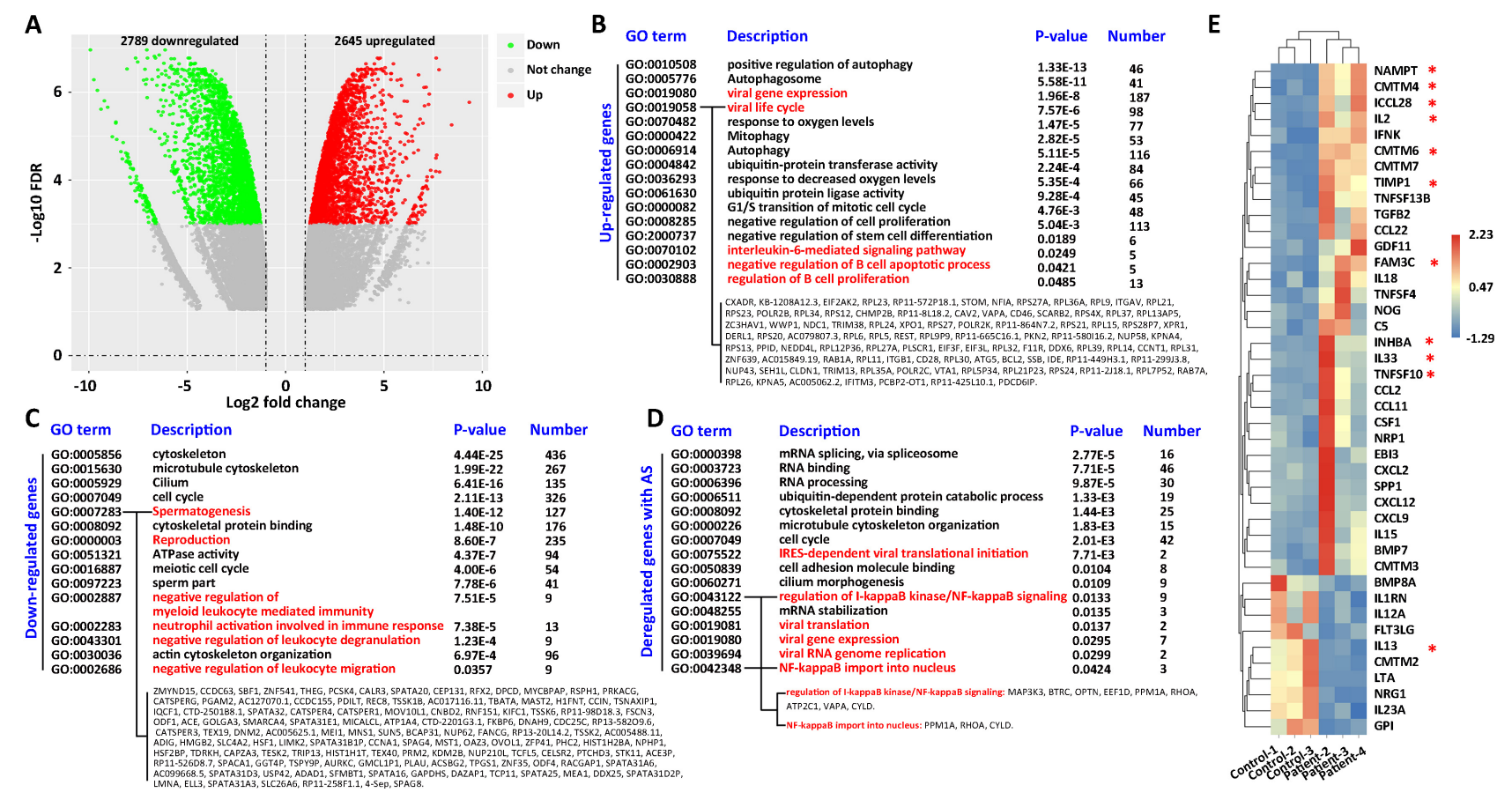

591 Figure 5. SARS-CoV-2 infection alters expression patterns of genes in testes of

592 patients with COVID-19. (A) Volcano plot showing the deregulated genes in testes of

593 COVID-19 patients compared to those controls. 2645 genes and 2789 genes were up-

594 regulated and down-regulated in COVID-19 patient testes, respectively. Significantly

595 deregulated genes have an FDR of $<0.001$ and fold change of $>2$. Three biological

596 replicates were indicated. (B-D) Gene ontology analyses of up-regulated genes (B), down-

597 regulated genes $(\mathbf{C})$, and alternative splicing changed genes (D) that identified in testes of

598 COVID-19 patients. Genes enriched in the viral life cycle (B), spermatogenesis (C)

599 indicated fly-out according to the degree of differences. (E) Heat-map is showing the 600 expression of the inflammatory cytokines in testes of COVID-19 patients using P601 value $<0.05$ as the cut-off. Genes significantly up-regulated or down-regulated using 602 FDR $<0.001$ labeled with asterisks. 
A

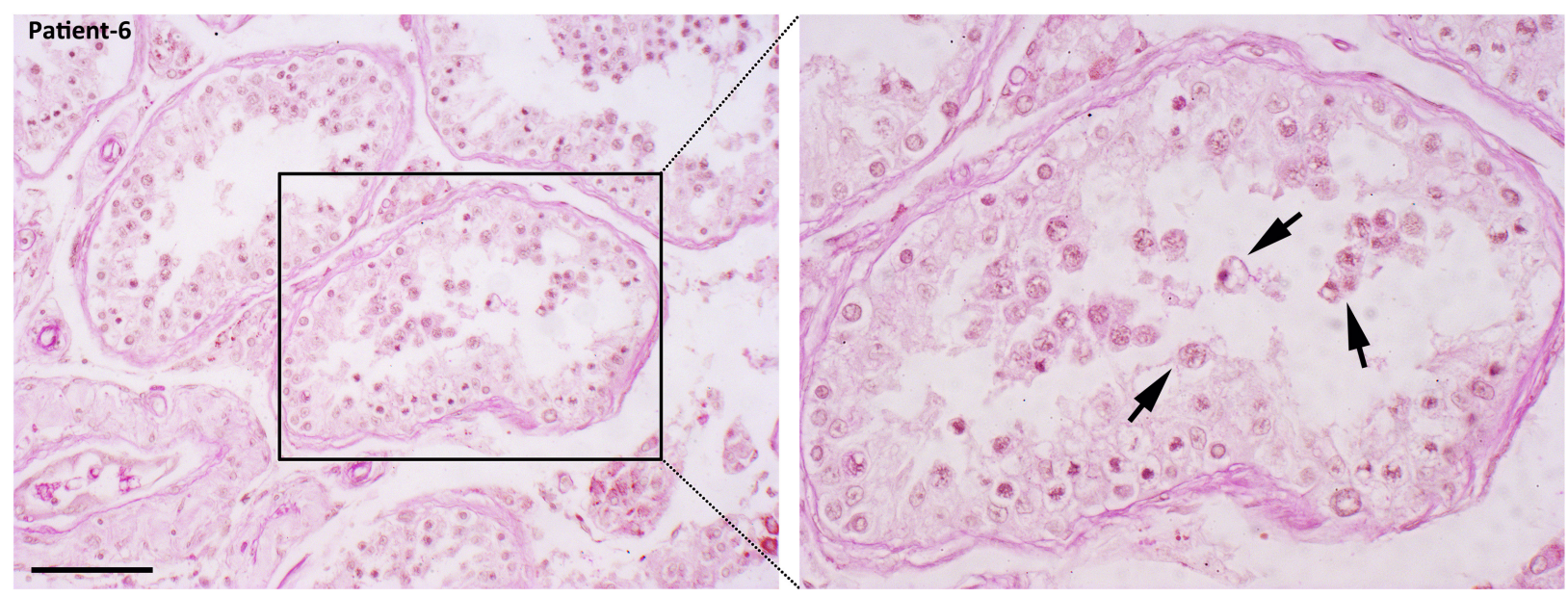

B

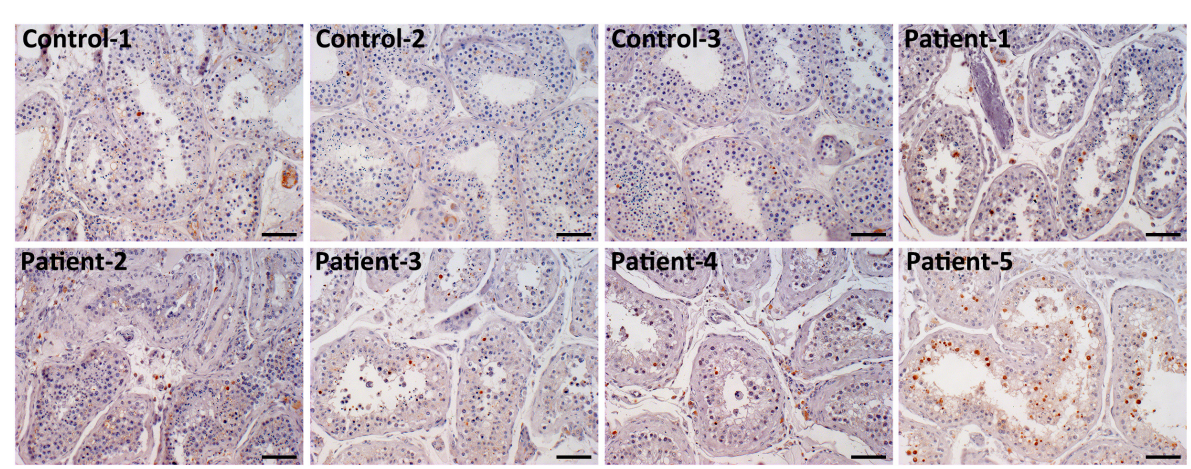

C

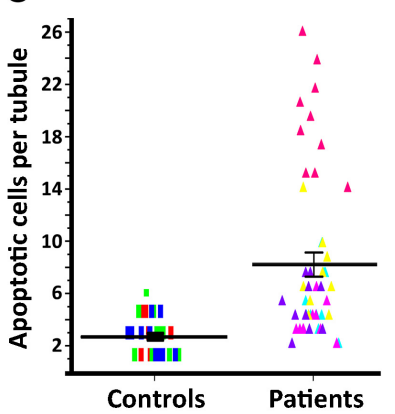

D

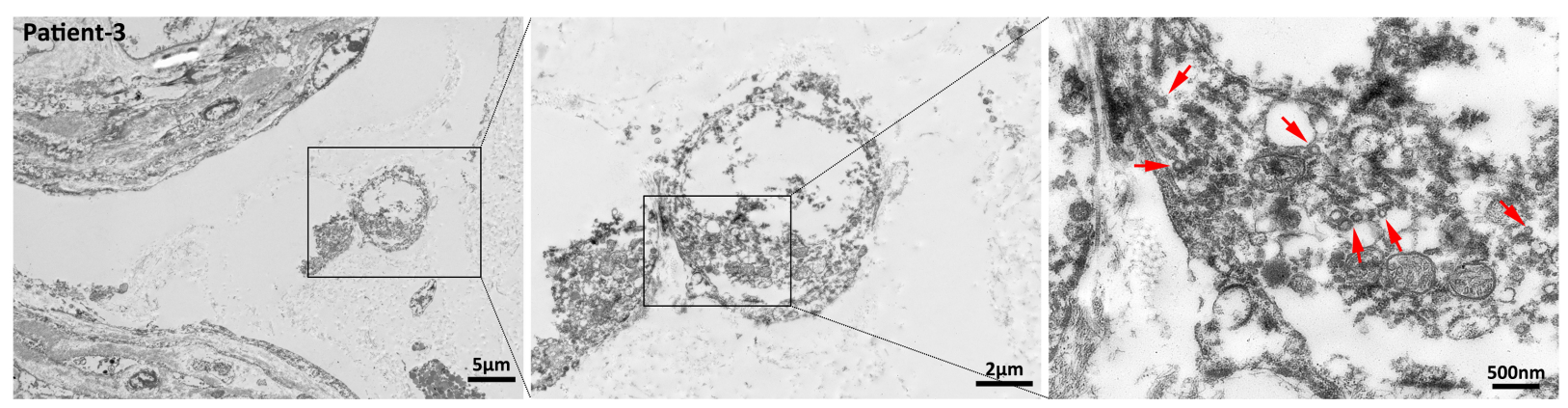

604 Fig.S1. (A) Representative PAS-staining images of Patient-6 testes are shown. (B) 605 Representative result of TUNEL assays is showing the staining for testicular sections of 606 control patients (control-1, 2, and 3) and COVID-19 patients (patient-1, 2, 3, 4, 5). Scale 607 bars $=100 \mu \mathrm{m}$. (C) The quantification of positive TUNEL cells in (B). (D) Electron microscopy 608 of the testis from Patient-3, showing coronavirus particles suggestive of viral infection (viral 609 particles highlighted by red arrows). 

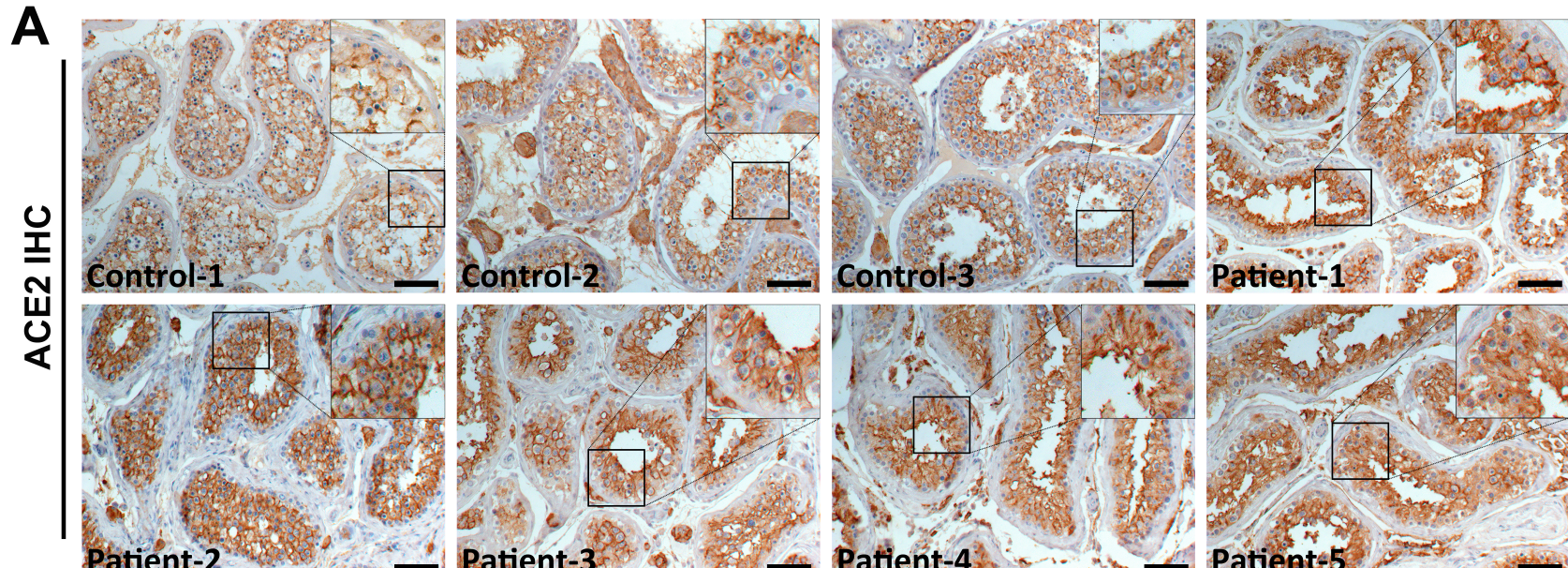

B
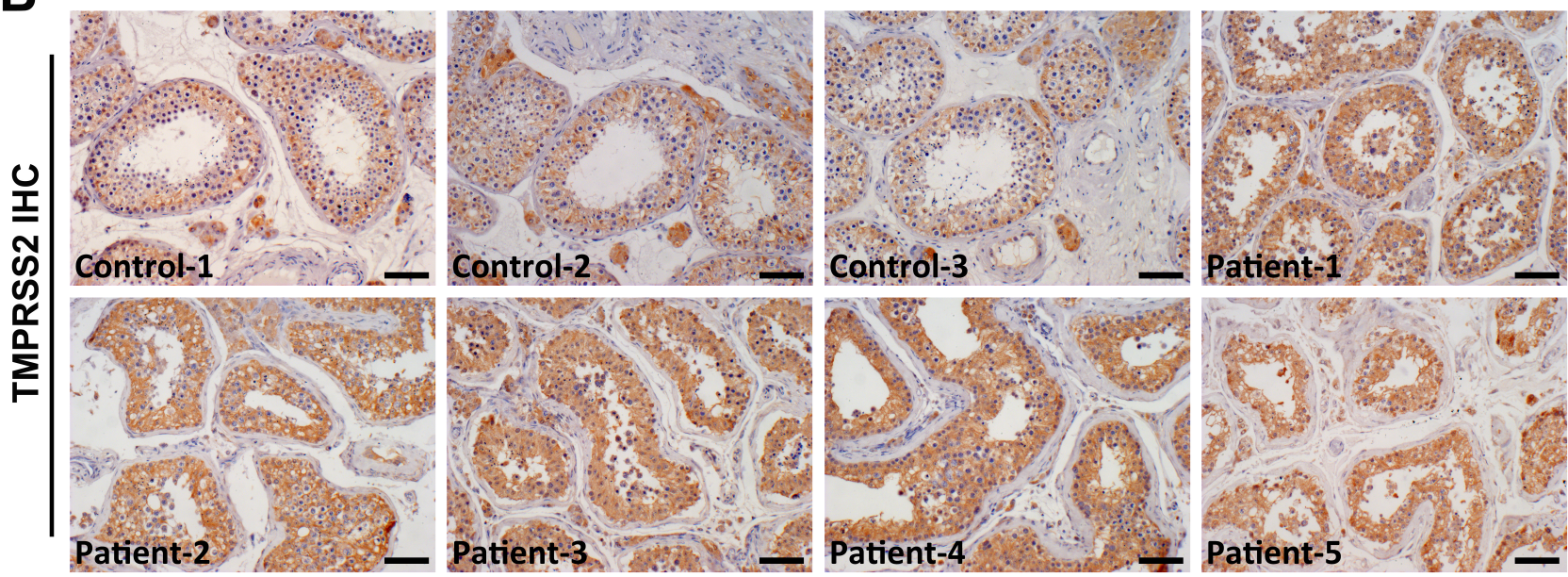

613 Fig.S2. Immunohistochemical stainings of ACE2 and TMPRSS2 in the testicular

614 sections are shown. (A) Representative ACE2 immunohistochemical (IHC) staining

615 images in the testicular sections of all control patients (control-1, 2, and 3) and COVID-19

616 patients (patient-1, 2, 3, 4, and 5) are shown. Scale bars=100 $\mu$ m. (B) Representative

617 TMPRSS2 immunohistochemical (IHC) staining images in the testicular sections of all

618 control patients (control-1, 2, and 3) and COVID-19 patients (patient-1, 2, 3, 4, and 5) are

619 shown. Scale bars $=100 \mu \mathrm{m}$. 
A

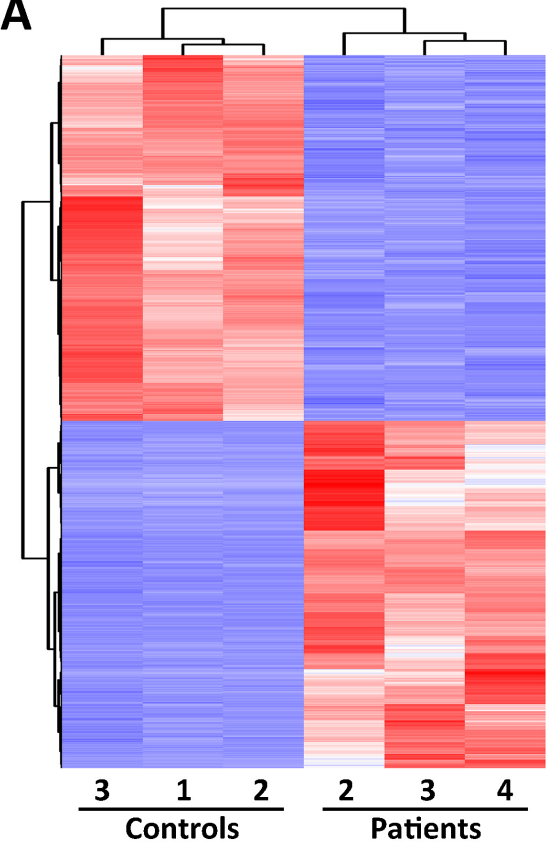

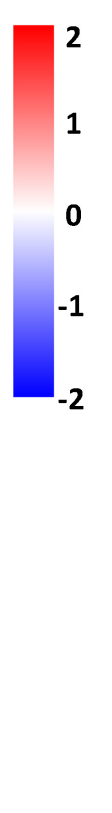

B

15

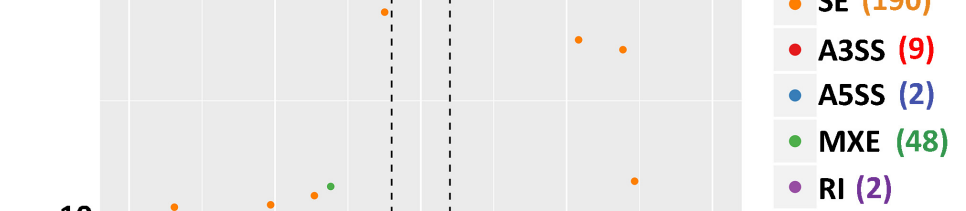

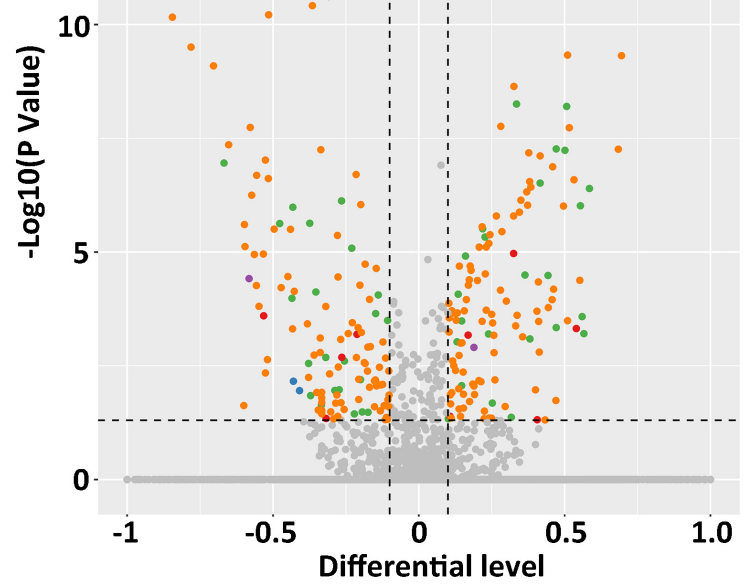

621 Fig.S3. RNA-seq analyses show differential gene expression and alternative splicing

622 events in the testes of COVID-19 patients. (A) Heat-map of genes significantly 623 deregulated (FDR<0.001 and fold change $>2$ ) in testes of COVID-19 patients compared to 624 those controls. (B) Volcano plot showing alternative splicing events of the deregulated 625 genes in testes of COVID-19 patients compared to those controls. Five types of alternative 626 splicing events are shown with different colors.

628 Table S1. The detection of nuclear acid of SARS-CoV-2 virus in FFPE testis samples of 629 COVID-19 patients by RT-qPCR.

630

631 Table S2. Patient clinical characteristics and medical history.

633 Table S3. The total coding and IncRNA genes are identified from the testes of control and 634 COVID-19 patients determined by RNA-seq analyses. 
636 Table S4. The up- and down-regulated genes are identified from testes of COVID-19 637 patients determined by RNA-seq analyses.

638

639 Table S5. 251 alternative splicing events are identified from deregulated genes determined 640 by RNA-seq. 


\section{Figures}
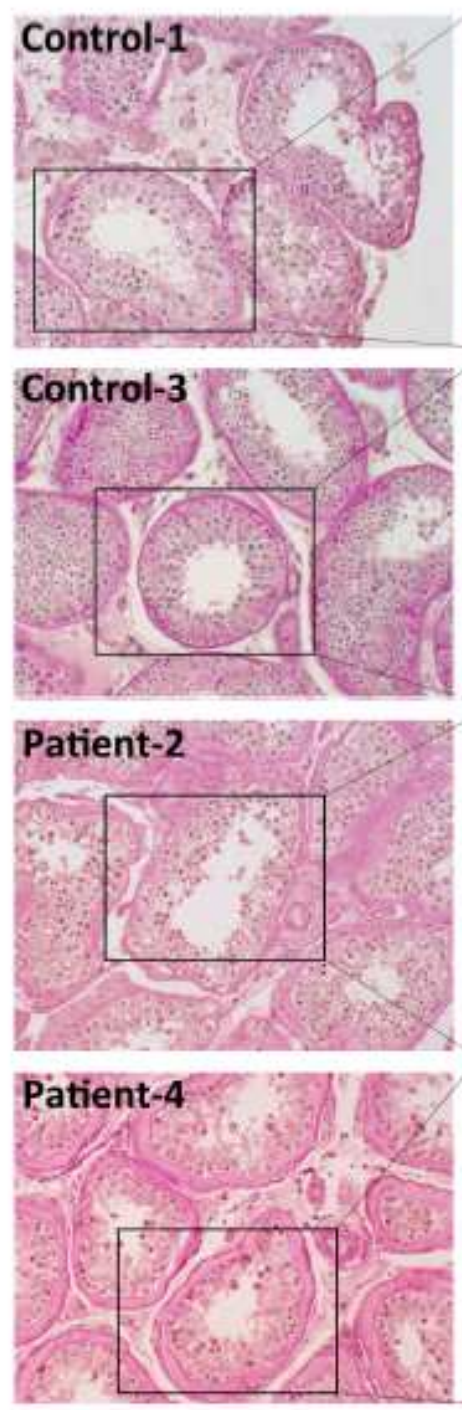
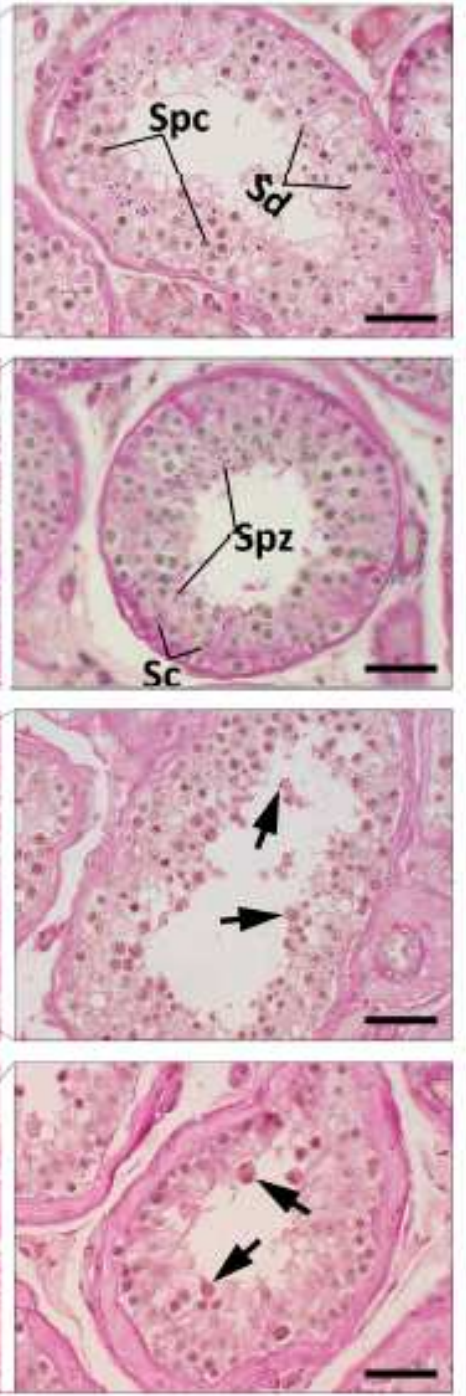
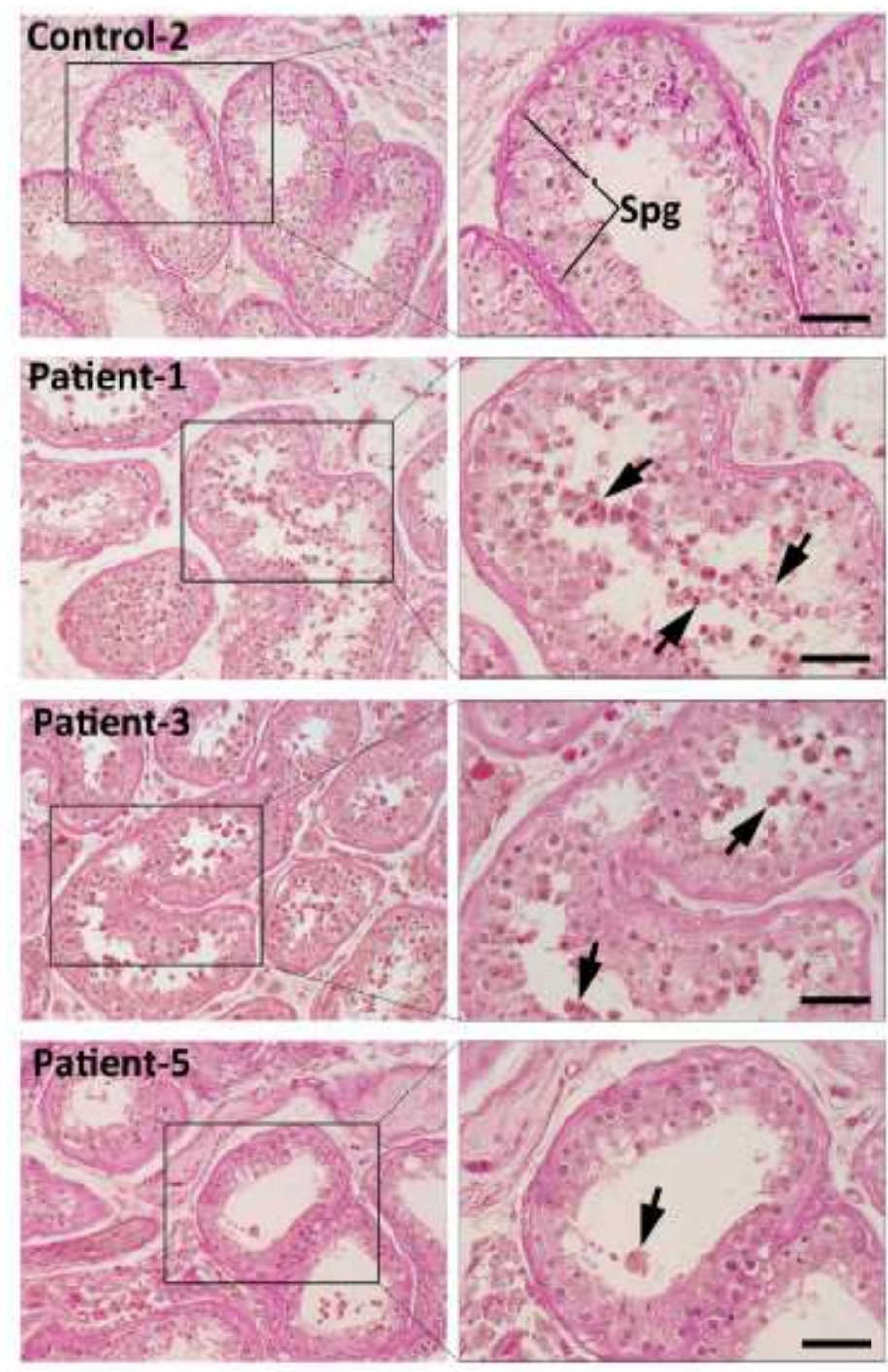

\section{Figure 1}

Spermatogenesis damages were observed in COVID-19 patients. Histological analyses of the testicular sections from COVID-19 patients (patient-1, 2, 3, 4, and 5) and uninfected control patients (control-1, 2, and 3 ) showing numerous degenerated germ cells fell off to the lumen in seminiferous tubules of all five COVID-19 patients, whereas normal spermatogenesis was observed in control patients. Spg, Spermatogonia; Spc, Spermatocytes; Sd, Spermatids; Spz, Spermatozoa; Sc, Sertoli cells. Arrows indicate degenerated germ cells. Scale bar $=100 \mu \mathrm{m}$. 

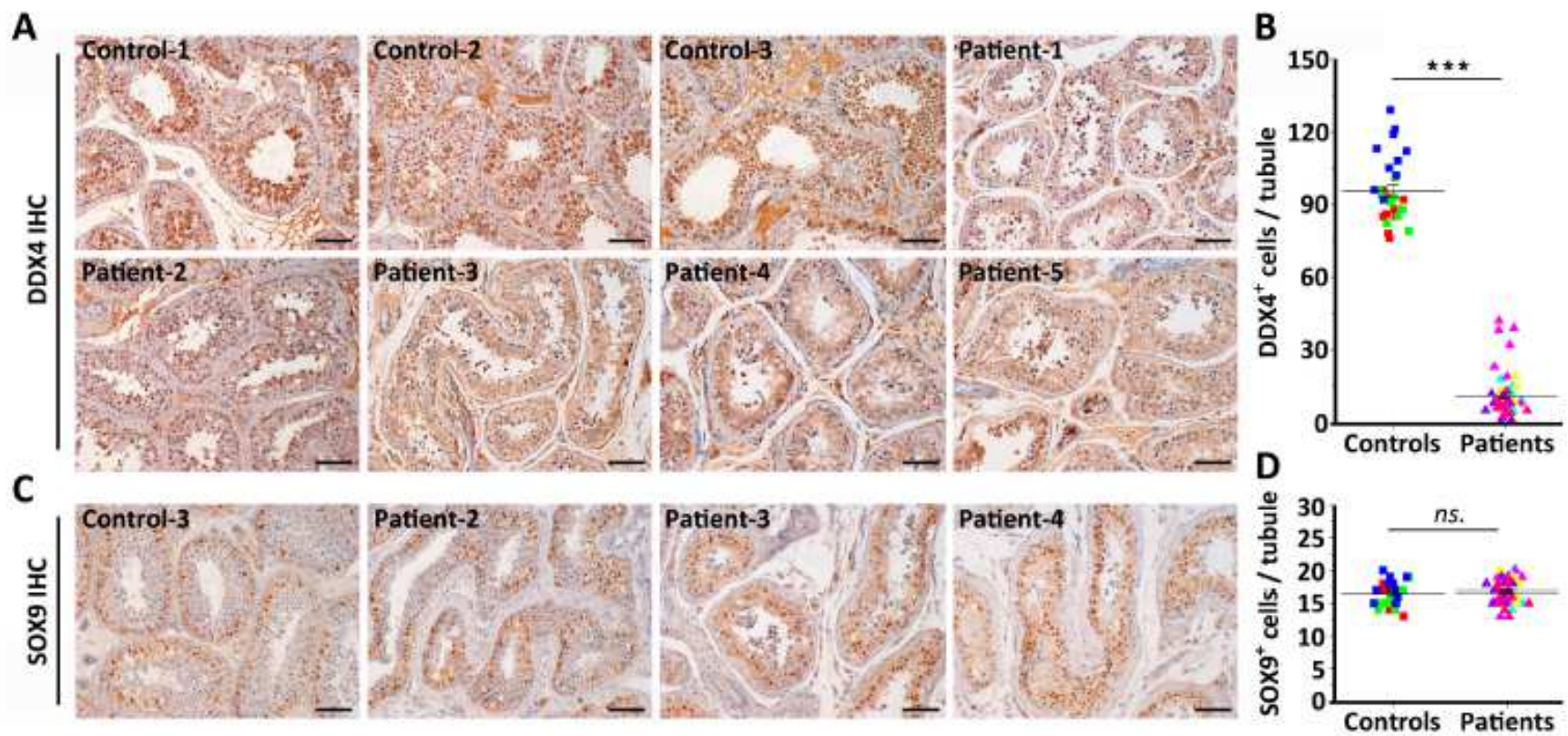

Figure 2

Germ cells but not Sertoli cells lost in the testes of COVID-19 patients. (A) Immunohistochemical staining of DDX4 (a germ cell marker) in the testicular sections of control patients (control-1, 2, and 3 ) and COVID19 patients (patient- $1,2,3,4$, and 5 ) is shown. Scale bar $=100 \mu \mathrm{m}$. (B) Scatter plot showing the quantification of DDX4-positive cell numbers per tubule in (A). ${ }^{\star \star \star}, P<0.001$ by Student's t-test. (C) Immunohistochemical staining of SOX9 (a Sertoli cell marker) in the testicular sections of control patient (control-3) and COVID-19 patients (patient-2, 3, and 4) is shown. Scale bar=100 $\mu \mathrm{m}$. (D) Scatter plot showing the quantification of SOX9-positive cell numbers per tubule in (C). ns., not significant. 


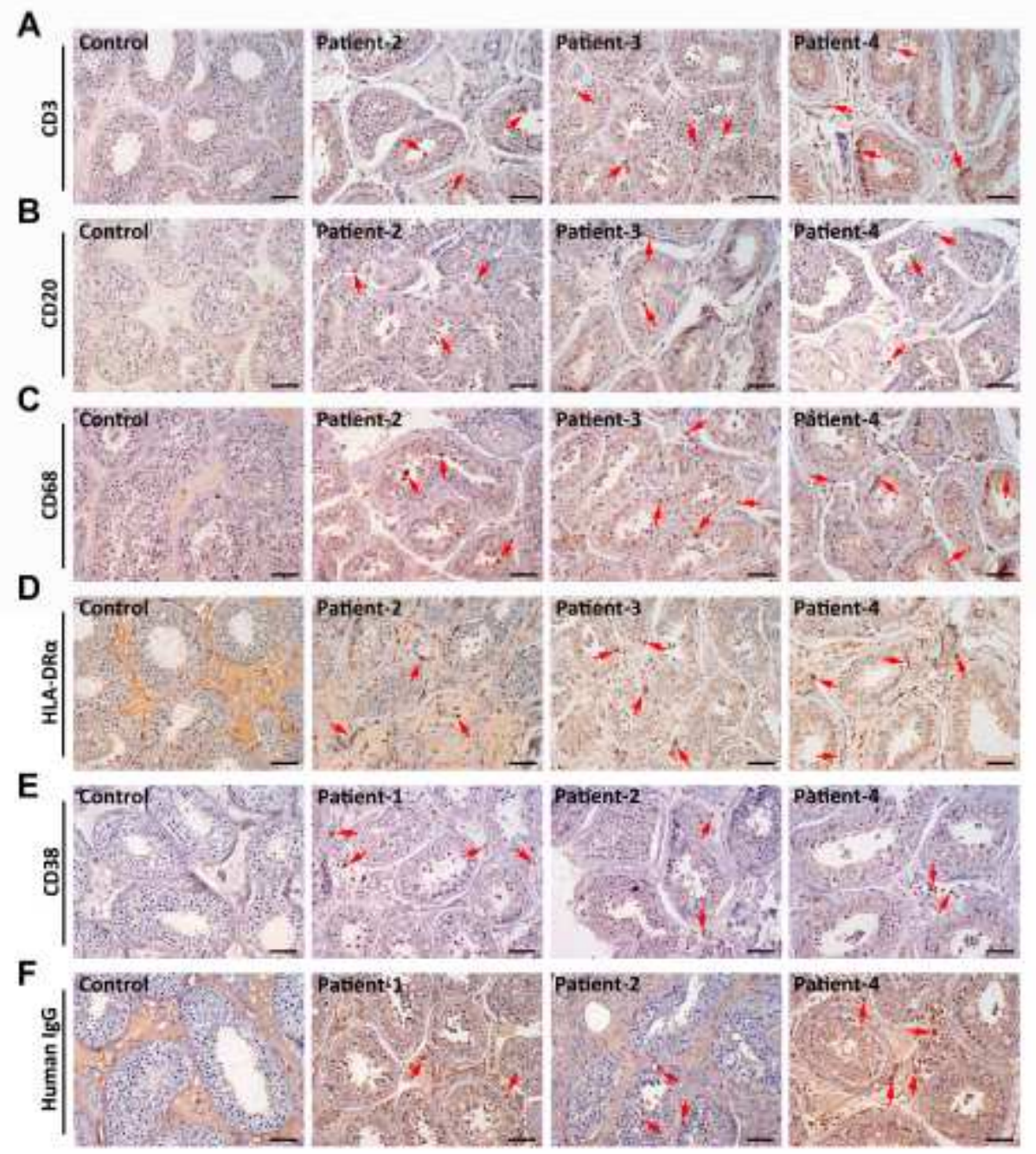

\section{Figure 3}

COVID-19 patient testes exhibit a prominent infiltration of various inflammatory cells. (A) Representative CD3 immunohistochemical staining images in the testicular sections of control and COVID-19 patients

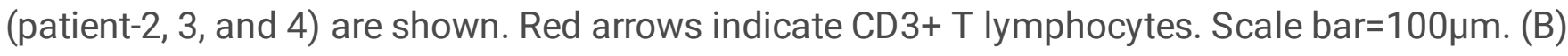
Representative CD20 immunohistochemical staining images in the testicular sections of control and COVID-19 patients (patient- 2, 3, and 4) are shown. Red arrows indicate CD20+ B lymphocytes. Scale bar $=100 \mu \mathrm{m}$. (C) Representative CD68 immunohistochemical staining images in the testicular sections of control and COVID-19 patients (patient-2, 3, and 4) are shown. Red arrows indicate CD68+ macrophages. Scale bar $=100 \mu \mathrm{m}$. (D) Representative HLA-DRa immunohistochemical staining images in the testicular sections of control and COVID-19 patients (patient-2, 3, and 4) are shown. Red arrows indicate HLA-DRA+ myeloid cells. Scale bar=100 $\mu \mathrm{m}$. (E) Representative CD38 immunohistochemical staining images in the testicular sections of control and COVID-19 patients (patient-1, 2, and 4) are shown. Red arrows indicate CD38+ cells. Scale bar $=100 \mu \mathrm{m}$. (F) Representative human IgG immunohistochemical staining images in the testicular sections of control and COVID-19 patients (patient-1, 2, and 4) are shown. Red arrows indicate IgG+ cells. Scale bar $=100 \mu \mathrm{m}$. 

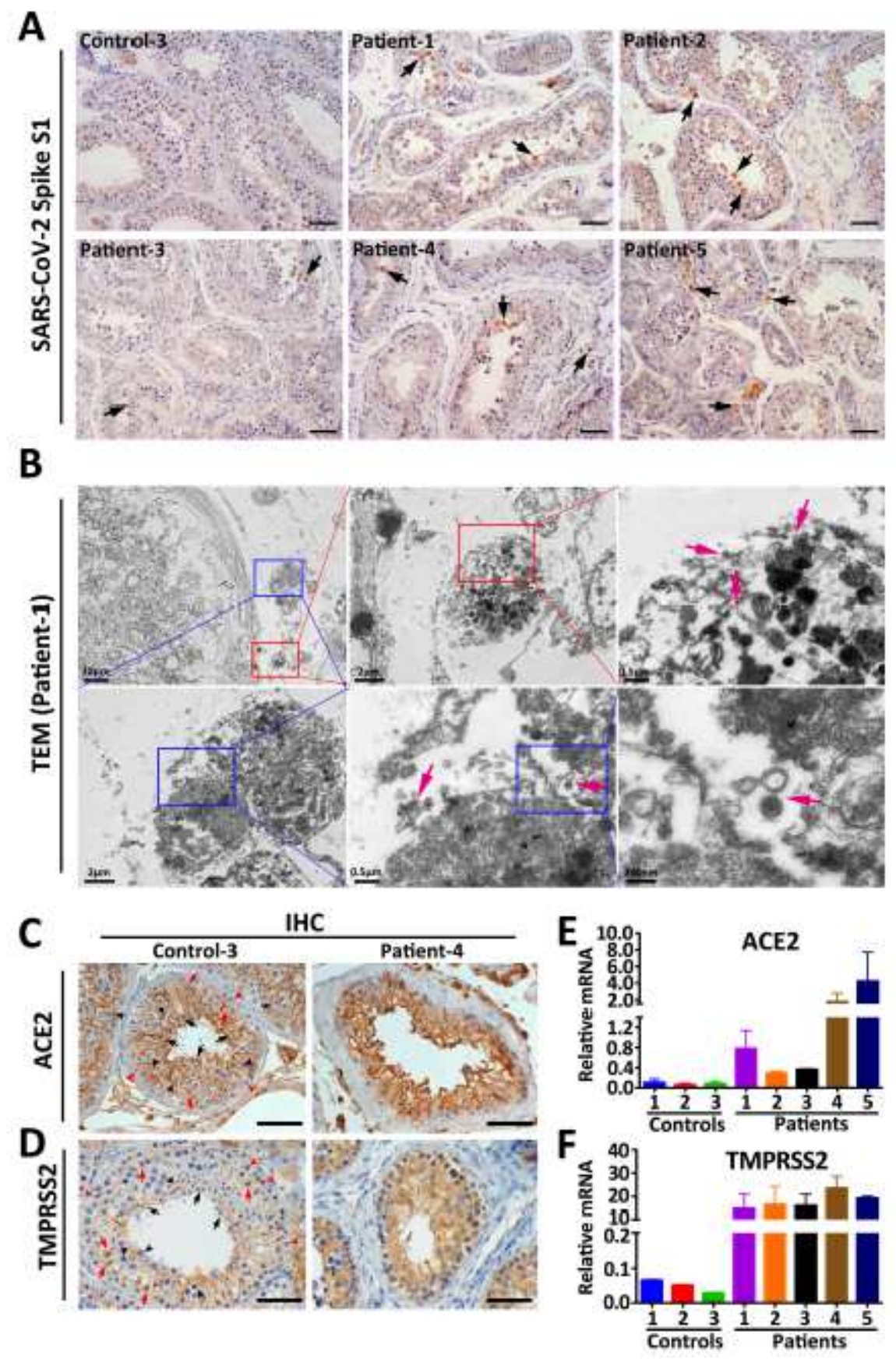

\section{Figure 4}

SARS-CoV-2 can invade testis and attack testicular 573 cells. (A) Representative SARS-CoV-2 Spike protein immunohistochemical staining images in the testicular sections of control (control-3) and COVID19 patients (patient-1, 2, 3, 4, and 5) are shown. Black arrows indicate SARS-CoV-2 Spike S1 positive cells. Scale bar $=100 \mu \mathrm{m}$. (B) Electron microscopy of the testis from Patient-1, showing coronavirus particles suggestive of viral infection (viral particles highlighted by red arrows). (C) Representative ACE2 immunohistochemical staining images in the testicular sections of control (control- 3) and COVID-19 patient (patient-4) are shown. Black arrows indicate round spermatids; Black arrowheads indicate pachytene spermatocytes; Red arrows indicate Sertoli cells; Red arrowheads indicate spermatogonia. Scale bar $=100 \mu \mathrm{m}$. (D) Representative TMPRSS2 immunohistochemical staining images in the testicular 
sections of control (control-3) and COVID-19 patient (patient-4) are shown. Black arrows indicate round spermatids; Black arrowheads indicate pachytene spermatocytes; Red arrows indicate Sertoli cells; Red arrowheads indicate spermatogonia. Scale bar $=100 \mu \mathrm{m}$. (E) RT-qPCR analyses of the relative ACE2 mRNA levels in control patients (control-1, 2, and 3) and COVID-19 patients (patient-1, 2, 3, 4, and 5). (F) RT-qPCR analyses of the relative TMPRSS2 mRNA levels in control patients (control-1, 2, and 3) and COVID-19 patients (patient-1, 2, 3, 4, and 5).

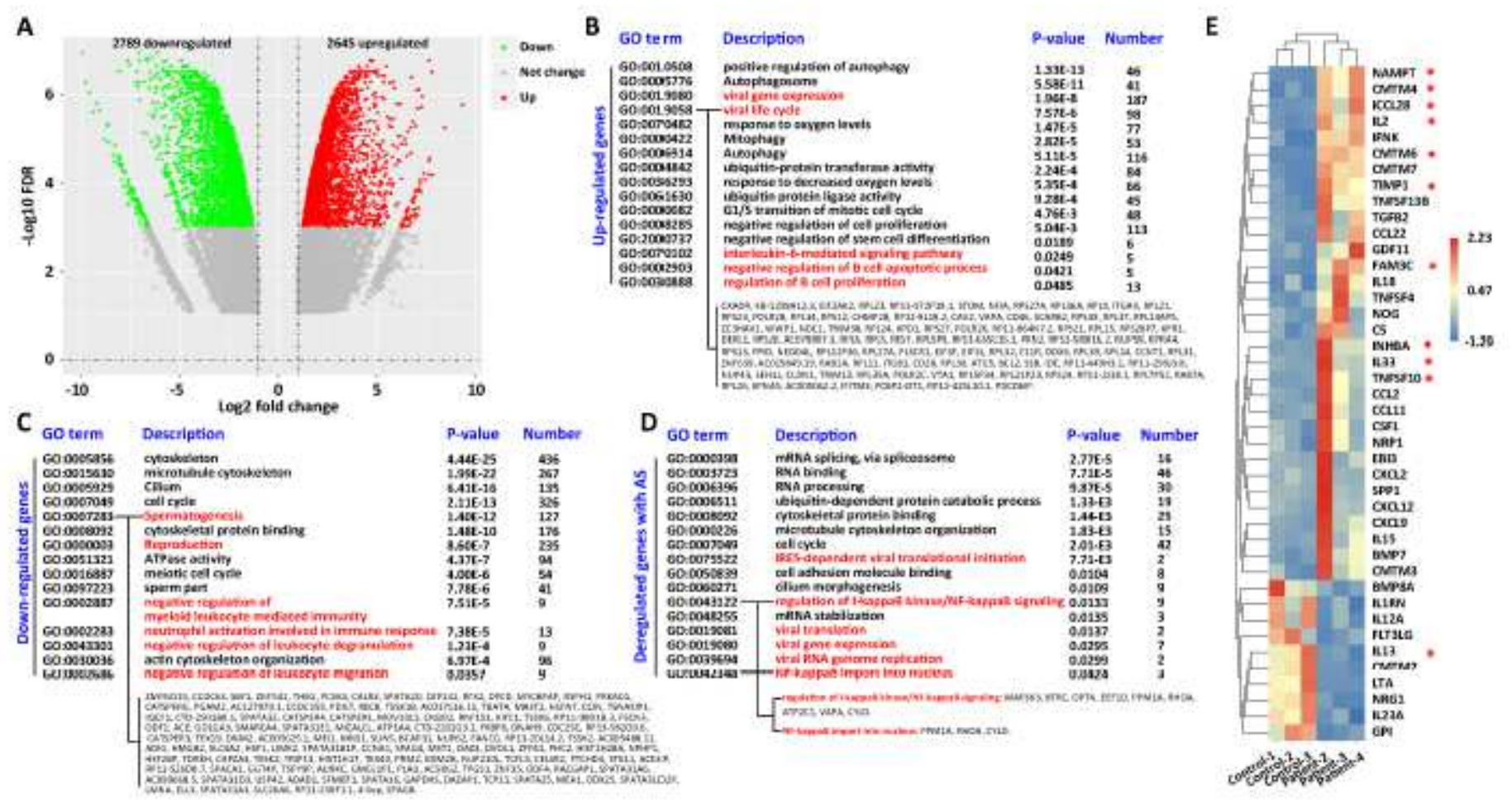

Figure 5

CoV-2 infection alters expression patterns of genes in testes of patients with COVID-19. (A) Volcano plot showing the deregulated genes in testes of COVID-19 patients compared to those controls. 2645 genes and 2789 genes were up regulated and down-regulated in COVID-19 patient testes, respectively. Significantly deregulated genes have an FDR of $<0.001$ and fold change of $>2$. Three biological replicates were indicated. (B-D) Gene ontology analyses of up-regulated genes (B), down regulated genes (C), and alternative splicing changed genes (D) that identified in testes of COVID-19 patients. Genes enriched in the viral life cycle (B), spermatogenesis (C) indicated fly-out according to the degree of differences. (E) Heat-map is showing the expression of the inflammatory cytokines in testes of COVID-19 patients using $P$ value $<0.05$ as the cut-off. Genes significantly up-regulated or down-regulated using FDR $<0.001$ labeled with asterisks.

\section{Supplementary Files}


This is a list of supplementary files associated with this preprint. Click to download.

- TableS3RPKMofALLGeneswithPvalue.xlsx

- TableS4Geneexprdiffwithanno.xlsx

- TableS5Junctioneventrecords.xlsx

- TableS1Patientclinical.docx

- TableS2Virusdetection.docx

- Figs1.png

- FigS2.png

- Figs3.png

- Tables1.png

- Tables2.png 